\title{
BENZIMIDAZOLE MOLECULE HYBRID WITH OXADIAZOLE RING AS ANTIPROLIFERATIVE AGENTS: IN-SILICO ANALYSIS, SYNTHESIS AND BIOLOGICAL EVALUATION
}

\author{
MOHAMMAD RASHID ${ }^{1 *}$, OBAID AFZAL ${ }^{2}$ AND ABDULMALIK SALEH ALFAWAZ ALTAMIMI ${ }^{2 *}$ \\ ${ }^{1}$ Department of Pharmaceutical Chemistry and Pharmacognosy, College of Dentistry and Pharmacy, Buraydah Colleges, Al-Qassim 31717, \\ Kingdom of Saudi Arabia. \\ ${ }^{2}$ Department of Pharmaceutical Chemistry, College of Pharmacy, Prince Sattam Bin Abdulaziz University, Al-Kharj-11942, Saudi Arabia.
}

\begin{abstract}
A new series of benzimidazole molecules hyride with oxadiazole ring were design with an intention to search new antiproliferative lead compound. In-Silico toxicity of lead compound was also performed by using T.E.S.T software tool, a program from US Environmental Protection Agency. Drug like properties and bioactivity score for drug targets of designed compounds were calculated by molinspiration tool and obtained result found to obey Lipinski's rule that indicates the compound are orally active molecules. Osiris property explorer was used for the prediction of drug relevant properties and toxicity of synthetic compounds. Pre ADMET server was also used to estimate ADME properties of synthetic compounds. Antiproliferative activities of compounds were investigated at the National Cancer Institute (NCI) against NCI 60 cell line panel, results showed good to notable anticancer activity. So that, these new hybrids compounds could serve as potential template to become leads in near future for the discovery and development of new effect orally drugs molecules. Two compounds, 4c[1-(1H-benzo[d] imidazol-2-yl)-3-(5-((4-methylpiperazin-1-yl)methyl)-1,3,4 -oxadiazol-2-yl) propan-1-one $]$ and $4 \mathbf{k}[3-((5-(3-(1 H$-benzo[ $d]$ imidazol-2-yl)-3oxopropyl)-1, 3, 4-oxadiazol-2-yl) methyl)-5-methylpyrimidine-2,4(1H,3H)-dione] were exhibited highest drug score and emerged as lead compounds and motivates for further development of more effective and safer compounds.
\end{abstract}

Keywords: Benzimidazole, Oxadiazole, Lipinski's rule, OSIRIS Property, Pre ADMET, T.E.S.T tool, NCI and Antiproliferative activity.

\section{INTRODUCTION}

Cancer is not only affects the health but also the economy of the patient. During the life of a healthy species tissue is constantly regenerated through cell death and cell division. It is essential for survival that a delicate balance between the two is kept. Damage of the genetic material can disrupt this balance which may give rise to abnormal growths. So that a term for disease in which abnormal cells divide mitosis without control (uncontrolled growth of cells) is called Cancer. Cancer cells can invade nearby tissues and spread through bloodstream and lymphatic system to other parts of the body (metastasis)[1-3]. In the present era, a large number of medications acting through different mechanisms for the treatment of cancer are available but the effectiveness of many existing medication is limited by their toxicity to normal rapidly growing cells and may develop resistance to that drug. Another drawback is that majority of the drugs currently in the market are not specific [4]. Therefore there is a substantial need to develop a new, most effective and less toxic anticancer agents.

Different classes of heterocyclic and fused heterocyclic compounds have been identified through molecular biology, empirical screening and rational drug development in search of anticancer agents [5-6]. Especially nitrogen containing heterocyclic systems like azoles nucleus playing a vital role in the discovering novel drugs with potential anticancer activity. In terms of searching it could be considered that the benzimidazole nucleuses are of great importance in their biological as well as synthetic approach of medicinal chemistry. Scientists from worldwide have reported remarkable anticancer/antitumor/antiproliferative [717], anti-inflammatory [11], antifungal [18], antioxidant [19], antiviral including anti-HIV [20], antibacterial [21] and cysticidal activities [22] of benzimidazole derivatives. Similarly oxadiazole is a class of heterocyclic compounds that have attracted significant interest in medicinal chemistry owing to their wide range of pharmacological activities including anticancer/antitumor/antiproliferative activities [23-26]. The present research work, involves the design, synthesis, characterization, in-silico analysis and in-vitro anticancer activity is based on foregoing background review literature described in Figure 1.

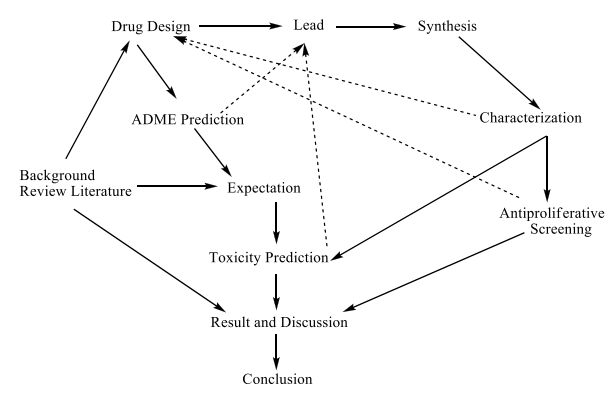

Figure 1. Research outline for the searching of new Antiproliferative agents.

We tried to shed some rational design target compounds from market approved anticancer drugs like Bendamustine (Treanda) and Chlorambucil having benzimidazole nucleus and other related heterocyclic moiety. Treanda is comprises of mechlorethamine and benzimidazole heterocyclic ring with a butyric acid substituent, a rationally design purine analog and alkylation hybrid of Chlorambucil [9] (Figure 2). It was approved by the FDA (U.S.) for the treatment of chronic lymphocytic leukemia (CLL) on 20 March 2008 and about 6 months later (31 October 2008), also approved for patients with indolent B-cell non-hodgkin's lymphoma (NHL) [27-28]. In view of these facts, it was thought worthwhile to design a rational template having benzimidazole residue as pharmacophoric group with butyric acid substituent and further clubbed with oxadiazole heterocyclic ring systems [9, 14] like Proxazole [29], IMC-094332 [30], Hoechst-33258 [31] and Nocodazol [32] having antitumor activity with a sight to produce potential anticancer agents (Figure 3).

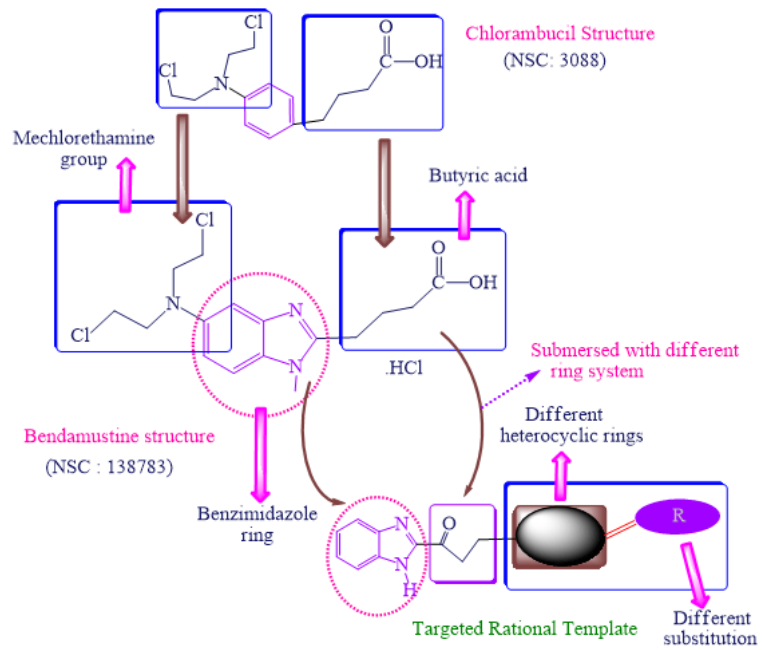

Figure 2. Rationally design and prepared a template for synthetic Scheme from marketed anticancer drug like Bendamustine and Chlorambucil.

Majority of the synthetic drugs fails because of inadequate information about their ADMET (absorption, distribution, metabolism, excretions and toxicity), Drug likeness, drug score and bioactivity profile. In view of these facts, it was thought worthwhile to design a rational template having benzimidazole residue as pharmacophoric group with butyric acid substituent and further clubbed with oxadiazole heterocyclic ring systems like Proxazole [29], IMC-094332 [30], 
Hoechst-33258 [31] and Nocodazol [32] having antitumor activity with a view to produce potential anticancer agents (Figure 3).

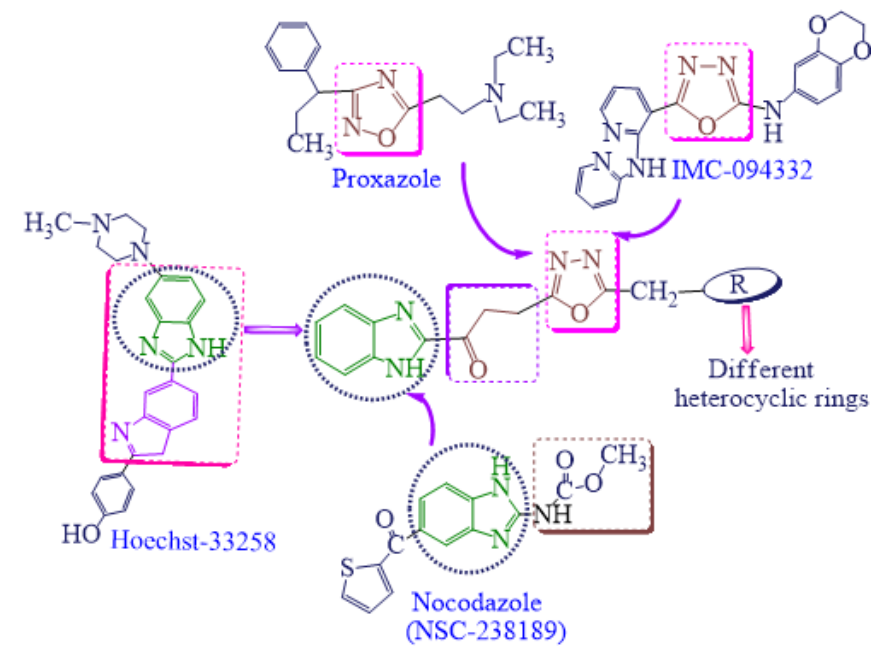

Figure 3. Rational template used for preparing synthetic derivatives.
A number of new hybrid molecules were synthesized and screened for their anticancer activities at the Development Therapeutic Program (DTP), National Cancer Institute (NCI), Chemotherapeutic Research Division, United States of America (USA) against full NCI 60 cell line panel representing on full nine human systems as Leukemia, Melanoma and cancers of Lung, Colon, Brain, Breast, Ovary, Kidney and Prostate according to their applied protocol. The synthetic scheme was carried out into the scientific microwave synthesizer (model No. CATA-R, Catalyst systems, India) and followed to prepare the title compounds. Modern analytical techniques (IR, NMR \& Mass) and elemental analysis were applied to establish the structure of the newly synthesized compounds.

The selected compounds were submitted to NCI and granted NCS codes as NCS: 759209(4a), NCS: 759210 (4b), NCS: 759211 (4c), NCS: 760445(4d), NCS: 759212 (4e), NCS: 755140(4f), NCS: 755141 (4g), NCS: 755142 (4h), NCS: 755143(4j), NCS: $761982(\mathbf{4 k})$ and NCS: 761983 (4l) for screening their anticancer activity against full NCI 60 cell line panel at the dose of $1 \times 10^{-5} \mathrm{M}$ (Table 1). Majority of the synthetic drugs fails because of inadequate information about their ADME/T (absorption, distribution, metabolism, excretions and toxicity), drug likeness, drug score and bioactivity profile. So that in the present study author design and synthesized new benzimidazole derivatives having oxadiazole ring and also predict their physiochemical property, ADMET, drug likeness, drug score, bioactivity profile and in-silico toxicity on the basis of Molinspiration, Pre ADMET prediction, Osiris property explorer and T.E.S.T tool respectively.

Table 1: Growth percent and mean growth percent of NCI cancer cell lines against the compounds at the dose of $1 \times 10^{-5} \mathrm{M}$, sensitivity and NSC: Code.

\begin{tabular}{|c|c|c|c|c|c|c|c|}
\hline Compd. & NSC: Code & The most sensitive cell line & $\begin{array}{l}\text { Growth \% of the most } \\
\text { sensitive cell line }\end{array}$ & $\begin{array}{c}\text { Range of } \\
\text { growth, \% }\end{array}$ & Mean & Range & Activity \\
\hline $4 a$ & 759209 & $\begin{array}{c}\text { HOP-92 } \\
\text { (Non-Small Cell Lung Cancer) }\end{array}$ & 61.48 & $61.48-119.43$ & 93.83 & 57.95 & active \\
\hline $4 \mathbf{b}$ & 759210 & $\begin{array}{c}\text { MOLT-4 } \\
\text { (Leukemia) } \\
\end{array}$ & 55.75 & $55.75-117.03$ & 88.91 & 61.28 & active \\
\hline $4 c$ & 759211 & $\begin{array}{c}\text { UO-31 } \\
\text { (Renal Cancer) }\end{array}$ & 68.81 & $68.81-137.83$ & 99.18 & 69.02 & active \\
\hline $4 d$ & 760445 & $\begin{array}{c}\text { EKVX } \\
\text { (Non-Small Cell Lung Cancer) }\end{array}$ & 67.58 & $67.58-148.14$ & 100.53 & 80.56 & active \\
\hline $4 e$ & 759212 & $\begin{array}{c}\text { UO-31 } \\
\text { (Renal Cancer) }\end{array}$ & 83.36 & $83.36-132.66$ & 103.89 & 49.30 & inactive \\
\hline $4 f$ & 755140 & $\begin{array}{c}\text { UO-31 } \\
\text { (Renal Cancer) }\end{array}$ & 84.84 & $84.84-129.33$ & 106.04 & 44.49 & inactive \\
\hline $4 \mathrm{~g}$ & 755141 & CCRF-CEM (Leukemia) & 28.46 & $28.46-125.48$ & 96.09 & 97.02 & active \\
\hline $4 h$ & 755142 & $\begin{array}{c}\text { SR } \\
\text { (Leukemia) }\end{array}$ & 82.01 & $82.01-126.78$ & 105.73 & 44.77 & inactive \\
\hline $4 \mathbf{i}$ & $\mathrm{Nt}$ & & & & & & \\
\hline $4 \mathbf{j}$ & 755143 & MALME-3M (Melanoma) & 77.82 & $77.82-123.77$ & 104.78 & 45.95 & inactive \\
\hline $4 k$ & 761982 & MDA-MB-435 (Melanoma) & -45.79 & $-45.79-90.70$ & 20.03 & 136.49 & active \\
\hline 41 & 761983 & MOLT-4 (Leukemia) & 8.54 & $8.54-104.24$ & 78.75 & 95.70 & active \\
\hline
\end{tabular}

Active compounds, which showed growth inhibition $\leq 32 \%$ for that particular cell lines, Percent cell growth reduction following $48 \mathrm{~h}$ incubation with test compounds (Sulphorhodamine B procedure), Negative numbers indicate the cell killed.

\section{MATERIAL AND METHODS}

\subsection{General information}

Melting points were taken on a liquid paraffin bath in open capillary tubes and are uncorrected. Progress of the reactions was monitored by using TLC plates (silica gel G), Toluene: Ethyl acetate: Formic acid (5:4:1, v/v/v) and Benzene: Acetone $(9: 1, \mathrm{v} / \mathrm{v})$ used as solvent systems. The spots were located by exposure to iodine vapors or under UV-light. Microwave irradiation of reactions was done in a scientific microwave synthesizer (model No. CATA-R, Catalyst systems). The chemicals (reagents and solvents) used for experimental work were commercially procured from various chemical unit like E. Merck Ltd., CDH, S.D. Fine chemicals and Qualigens. These solvent and reagents were of LR grade and purified before use. Nuclear magnetic resonance ( $\left.{ }^{1} \mathrm{H}-\mathrm{NMR} \&{ }^{13} \mathrm{C}-\mathrm{NMR}\right)$ spectra were recorded on Bruker spectrops in DPX-300 $\mathrm{MHz}$ in
DMSO- $d_{6} / \mathrm{CDCl}_{3}$; chemical shift $(\delta)$ values reported in parts per million $(\mathrm{ppm})$ and coupling constants $(J)$ in $H z$ using tetramethylsilane as internal reference. The splitting pattern abbreviations are as follows: s, singlet; bs, broad singlet; $\mathrm{d}$, doublet; dd, double doublet; $\mathrm{t}$, triplet; q, quadruplet; $\mathrm{m}$, multiplet. The exchangeable protons $(\mathrm{OH}$ and $\mathrm{N} H)$ confirmed by the addition of $\mathrm{D}_{2} \mathrm{O}$. Mass spectra were recorded on LCMS/MS (Perkin-Elmer and LABINDIA, Applied Biosystem, Mass Lynx version 4.1) spectrometer, model no API 3000 and presented as $m / z$. The $m / z$ values of the more intense peaks are mentioned. The Fourier Transform Infra Red (FTIR) spectra recorded on FT/IR (Jasco, Japan), model no. 410 , using $\mathrm{KBr}$ pellets; $v_{\max }$ values are given in $\mathrm{cm}^{-1}$. Elemental analyses performed on a Perkin-Elmer 240 analyzer and found in the range of $\pm 0.4 \%$ for each element analyzed (C, H \& N). The synthetic compounds were purified by recrystallization with suitable solvent and found pure upon TLC examination. 


\subsection{Synthesis}

Compounds (4a-l) were synthesized by the following steps as per Scheme $\mathbf{1}$.

\subsubsection{Synthesis of 4-(1H-benzo[d]imidazol-2-yl)-4-oxobutanoic acid (1)}

A solution of $o$-phenylenediamine $(0.01 \mathrm{~mol}), \alpha$-ketoglutaric acid (equimolar; $0.01 \mathrm{~mol}) \mathrm{in}$ methanol/water mixture $(1: 1)$ and $\mathrm{HCl}(4 \mathrm{~N} ; 5 \mathrm{~mL})$ was refluxed for $6 \mathrm{~h}$ and then left to cool to room temperature. The $\mathrm{NaOH}$ solution $(10 \% \mathrm{w} / \mathrm{v})$ was added slowly to neutralize the reaction mixture, a solid mass precipitated out, which was filtered, washed with water and recrystallized with ethanol. It gave effervescence when treated with sodium bicarbonate. Yield: 87\%; Mp. 261$262^{\circ} \mathrm{C} ; \mathrm{R}_{\mathrm{f}}=0.71(\mathrm{~T}: \mathrm{E}: \mathrm{F}) . \mathrm{IR}\left(\mathrm{KBr}, \mathrm{cm}^{-1}\right): 3394(\mathrm{O}-\mathrm{H}), 3326(\mathrm{~N}-\mathrm{H}), 3114(\mathrm{C}-\mathrm{H}, \mathrm{Ar}-$ $\mathrm{H}), 2972\left(\mathrm{C}-\mathrm{H}, \mathrm{CH}_{2}\right), 1728(\mathrm{C}=\mathrm{O}), 1600(\mathrm{C}=\mathrm{N}), 1562(\mathrm{C}=\mathrm{C}) .{ }^{1} \mathrm{H}-\mathrm{NMR}$ (DMSO$\left.d_{6}\right): \delta 12.97\left(\mathrm{~s}, 1 \mathrm{H}, \mathrm{OH}, \mathrm{D}_{2} \mathrm{O}\right.$ exchangeable $), 12.34\left(\mathrm{~s}, 1 \mathrm{H}, \mathrm{NH}, \mathrm{D}_{2} \mathrm{O}\right.$ exchangeable), 7.66(d, $1 \mathrm{H}, J=7.5 \mathrm{~Hz}, \mathrm{H}-4$, benzimidazole $), 7.48(\mathrm{t}, 1 \mathrm{H}, \quad J=$ $7.2 \mathrm{~Hz}, \mathrm{H}-7$, benzimidazole), 7.27(t, $2 \mathrm{H}, J=8.7 \mathrm{~Hz}, \mathrm{H}-5,6$, benzimidazole), $3.05\left(\mathrm{t}, 2 \mathrm{H}, J=6.9 \mathrm{~Hz}, \mathrm{CH}_{2}\right), 2.76\left(\mathrm{t}, 2 \mathrm{H}, J=6.9 \mathrm{~Hz}, \mathrm{CH}_{2}\right) .{ }^{13} \mathrm{C}-\mathrm{NMR}$ (DMSO- $\left.d_{6}\right)$ : $\delta 175.57(\mathrm{C}=\mathrm{O}), 168.21(\mathrm{C}=\mathrm{O}, \mathrm{COOH}), 159.67(\mathrm{C}=\mathrm{N}), 138.27,132.21,130.19$, 128.57, 124.65, 123.64(Ar-C), 34.23( $\left.\mathrm{CH}_{2}, \mathrm{CH}_{2} \mathrm{CO}\right), 31.36\left(\mathrm{CH}_{2}, \mathrm{CH}_{2} \mathrm{COOH}\right)$. ESI-MS (m/z): $218\left(\mathrm{M}^{+}\right)$. Anal. calcd. for $\mathrm{C}_{11} \mathrm{H}_{10} \mathrm{~N}_{2} \mathrm{O}_{3}$ : C, 60.55; H, 4.62; N, 12.84. Found: C, 60.63; H, 4.65; N, 12.98 .

\subsubsection{Synthesis of ethyl-4-(1H-benzo[d]imidazol-2-yl)-4-oxobutanoate (2)}

A solution of compound (1) $(0.01 \mathrm{~mol})$ in absolute ethanol $(25 \mathrm{~mL})$ was refluxed for $10 \mathrm{~h}$ in presence of conc. $\mathrm{H}_{2} \mathrm{SO}_{4}(0.1 \mathrm{~mL})$. After completion of the reaction, it was cooled to room temperature, diluted with cold water and then neutralized with $\mathrm{NaHCO}_{3}$ topH 7. A precipitate formed which was filtered and crystallized from ethanol. Yield: $81 \%$; Mp. $253-254^{\circ} \mathrm{C} ; \mathrm{R}_{\mathrm{f}}=0.60$ (T:E:F). IR $\left(\mathrm{KBr}, \mathrm{cm}^{-1}\right): 3330(\mathrm{~N}-\mathrm{H}), 3047(\mathrm{C}-\mathrm{H}, \mathrm{Ar}-\mathrm{H}), 2958\left(\mathrm{C}-\mathrm{H}, \mathrm{CH}_{2}\right), 1718(\mathrm{C}=\mathrm{O})$, $1640(\mathrm{C}=\mathrm{N}), \quad 1570(\mathrm{C}=\mathrm{C}) .{ }^{1} \mathrm{H}-\mathrm{NMR} \quad\left(\mathrm{DMSO}-d_{6}\right): \delta 12.51\left(\mathrm{bs}, 1 \mathrm{H}, \quad \mathrm{NH}, \mathrm{D}_{2} \mathrm{O}\right.$ exchangeable), 7.73(d, $1 \mathrm{H}, J=7.8 \mathrm{~Hz}, \mathrm{H}-4$, benzimidazole), $7.54(\mathrm{t}, 1 \mathrm{H}, J=$ $7.5 \mathrm{~Hz}, \mathrm{H}-7$, benzimidazole), $7.12(\mathrm{t}, 2 \mathrm{H}, J=7.5 \mathrm{~Hz}, \mathrm{H}-5,6$, benzimidazole), $3.82\left(\mathrm{q}, 2 \mathrm{H}, J=5.4 \mathrm{~Hz}, \mathrm{CH}_{2}, \mathrm{CH}_{2} \mathrm{CH}_{3}\right), 3.03\left(\mathrm{t}, 2 \mathrm{H}, J=7.2 \mathrm{~Hz}, \mathrm{CH}_{2}, \mathrm{CH}_{2} \mathrm{CO}\right)$, 2.71(t, $\left.2 \mathrm{H}, J=7.2 \mathrm{~Hz}, \mathrm{CH}_{2}, \mathrm{CH}_{2} \mathrm{CO}-\mathrm{O}\right), 1.15\left(\mathrm{t}, 3 \mathrm{H}, \mathrm{J}=1.2 \mathrm{~Hz}, \mathrm{CH}_{3}\right) .{ }^{13} \mathrm{C}-\mathrm{NMR}$ $\left(\mathrm{DMSO}-d_{6}\right): \delta 177.61(\mathrm{C}=\mathrm{O}), 165.37(\mathrm{C}=\mathrm{O}$, ester $), 156.47(\mathrm{C}=\mathrm{N}), 138.32,137.93$, $129.13,128.17,124.55,123.74(\mathrm{Ar}-\mathrm{C}), 69.07\left(\mathrm{CH}_{2}\right.$, ester), 30.81( $\left.\mathrm{CH}_{2}, \mathrm{CH}_{2} \mathrm{CO}\right)$, 28.36 $\left(\mathrm{CH}_{2}, \mathrm{CH}_{2} \mathrm{COO}\right), 20.31\left(\mathrm{CH}_{3}\right)$. ESI-MS $(\mathrm{m} / \mathrm{z}): 246\left(\mathrm{M}^{+}\right)$. Anal.calcd. for $\mathrm{C}_{13} \mathrm{H}_{14} \mathrm{~N}_{2} \mathrm{O}_{3}: \mathrm{C}, 63.40 ; \mathrm{H}, 5.73 ; \mathrm{N}, 11.38$. Found: $\mathrm{C}, 62.77 ; \mathrm{H}, 5.81 ; \mathrm{N}, 11.35$. (3)

2.2.3. Synthesis of 4-(1H-benzo[d]imidazol-2-yl)-4-oxobutane hydrazide

A solution of compound $2(0.01 \mathrm{~mol})$ and hydrazine hydrate $(0.015 \mathrm{~mol})$ in ethanol $(25 \mathrm{~mL})$ was refluxed for $8 \mathrm{~h}$. The reaction mixture was cooled, a solid precipitate separated out, which was filtered, dried and recrystallized from methanol. Yield: $85 \%$; Mp. $241-242^{\circ} \mathrm{C} ; \mathrm{R}_{\mathrm{f}}=0.53\left(\mathrm{~T}\right.$ : E: F). IR $\left(\mathrm{KBr}, \mathrm{cm}^{-1}\right)$ : 3494(N-H, NH $\left.\mathrm{N}_{2}\right), 3359(\mathrm{~N}-\mathrm{H}, \mathrm{NH}), 3151(\mathrm{C}-\mathrm{H}, \mathrm{Ar}-\mathrm{H}), 2947\left(\mathrm{C}-\mathrm{H}, \mathrm{CH}_{2}\right), 1691$ $(\mathrm{C}=\mathrm{O}), 1643(\mathrm{C}=\mathrm{N}), 1600(\mathrm{C}=\mathrm{C}) .{ }^{1} \mathrm{H}-\mathrm{NMR}\left(\mathrm{DMSO}-d_{6}\right): \delta 11.51(\mathrm{~s}, 1 \mathrm{H}, \mathrm{NH}$, benzimidazole, $\mathrm{D}_{2} \mathrm{O}$ exchangeable $), 10.72\left(\mathrm{~s}, 1 \mathrm{H}, \mathrm{NH}\right.$, hydrazide, $\mathrm{D}_{2} \mathrm{O}$ exchangeable), $9.05\left(\mathrm{bs}, 2 \mathrm{H}, \mathrm{NH}_{2}, \mathrm{D}_{2} \mathrm{O}\right.$ exchangeable), $7.68(\mathrm{~d}, 1 \mathrm{H}, J=7.8 \mathrm{~Hz}, \mathrm{H}-$ 4, benzimidazole), $7.50(\mathrm{t}, 1 \mathrm{H}, J=7.2 \mathrm{~Hz}, \mathrm{H}-7$, benzimidazole $), 7.27(\mathrm{t}, 2 \mathrm{H}, J=$ $7.5 \mathrm{~Hz}, \mathrm{H}-5,6$, benzimidazole), $3.02\left(\mathrm{t}, 2 \mathrm{H}, J=7.5 \mathrm{~Hz}, \mathrm{CH}_{2} \mathrm{CO}\right), 2.52(\mathrm{t}, 2 \mathrm{H}, J=$ $7.8 \mathrm{~Hz}, \mathrm{CH}_{2}$, hydrazide). ${ }^{13} \mathrm{C}-\mathrm{NMR}$ (DMSO- $\left.d_{6}\right): \delta 179.15(\mathrm{C}=\mathrm{O}), 162.72(\mathrm{C}=\mathrm{O}$, hydrazide), $156.32(\mathrm{C}=\mathrm{N}), 133.99,132.28,129.59,128.45,122.92,116.85$ (ArC), 30.17 $\left(\mathrm{CH}_{2}, \mathrm{CH}_{2} \mathrm{CO}\right), 22.27\left(\mathrm{CH}_{2}\right.$, hydrazide). ESI-MS $(\mathrm{m} / \mathrm{z}): 232\left(\mathrm{M}^{+}\right)$. Anal.calcd. for $\mathrm{C}_{11} \mathrm{H}_{12} \mathrm{~N}_{4} \mathrm{O}_{2}: \mathrm{C}, 56.89 ; \mathrm{H}, 5.21 ; \mathrm{N}, 24.12$. Found: C, 56.73; H, $5.27 ; \mathrm{N}, 24.15$.

2.2.4. Synthesis of 1-(1H-benzo[d]imidazol-2-yl)-3-(5-(chloromethyl)1,3,4-oxadiazol-2-yl) propan-1-one (4)

A mixture of 4-(1H-benzo[ $[$ ] imidazol-2-yl)-4-oxobutanehydrazide $3(0.001$ $\mathrm{mol}$ ) and chloro acetic acid (equimolar; $0.001 \mathrm{~mol}$ ) and $\mathrm{POCl}_{3}(5 \mathrm{~mL})$ was placed into a microwave reaction vessel equipped with a magnetic stir bar. The reaction vessel was kept into the scientific microwave synthesizer and irradiated at a power level of $6(60 \%, 420 \mathrm{~W})$ for $6-13 \mathrm{~min}$. After completion of the reaction, checked by single spot TLC using the solvent systems; Toluene: Ethyl acetate: Formic acid (5:4:1) or Benzene: Acetone (9:1), the reaction mixture was cooled and poured slowly onto crushed ice, neutralized with sodium bicarbonate solution. A solid mass precipitated out, which was filtered, washed with excess quantity of water to remove the inorganic component and then recrystallized with carbinol to get the pure compounds (4). Yield: $85 \%$; Mp. $223-224^{\circ} \mathrm{C} ; \mathrm{R}_{\mathrm{f}}=$ 0.65(T.E.F.). IR ( $\left.\mathrm{KBr}, \mathrm{cm}^{-1}\right)$ : 3350(N-H), 3020(C-H, Ar-H), 2935(C-H, $\left.\mathrm{CH}_{2}\right)$, 1710 $(\mathrm{C}=\mathrm{O}), 1682(\mathrm{C}=\mathrm{N}), 1574(\mathrm{C}=\mathrm{C}), 1304(\mathrm{~N}-\mathrm{N}=\mathrm{C}), 1164(\mathrm{C}-\mathrm{O}-\mathrm{C}$ asymmetric $)$, 1028 (C-O-C, symmetric), 714(C-Cl). ${ }^{1} \mathrm{H}-\mathrm{NMR}\left(\mathrm{CDCl}_{3}\right): \delta 12.51\left(\mathrm{~s}, 1 \mathrm{H}, \mathrm{NH}, \mathrm{D}_{2} \mathrm{O}\right.$ exchangeable), $7.80(\mathrm{~d}, 1 \mathrm{H}, J=7.8 \mathrm{~Hz}, \mathrm{H}-4$, benzimidazole $), 7.48(\mathrm{t}, 1 \mathrm{H}, J=$ $7.2 \mathrm{~Hz}, \mathrm{H}-7$, benzimidazole), 7.33(t, $2 \mathrm{H}, J=5.4 \mathrm{~Hz}, \mathrm{H}-5,6$, benzimidazole), $4.39\left(\mathrm{~s}, 2 \mathrm{H}, \mathrm{CH}_{2} \mathrm{Cl}\right), 3.29\left(\mathrm{t}, 2 \mathrm{H}, J=6.9 \mathrm{~Hz}, \mathrm{CH}_{2}\right), 2.90\left(\mathrm{t}, 2 \mathrm{H}, J=6.6 \mathrm{~Hz}, \mathrm{CH}_{2}\right.$, $\left.\mathrm{CH}_{2} \mathrm{CO}\right) .{ }^{13} \mathrm{C}-\mathrm{NMR}\left(\mathrm{CDCl}_{3}\right): \delta 169.45(\mathrm{C}=\mathrm{O}), 157.21,155.36(2 \mathrm{C}$, oxadiazole $)$, $153.67(\mathrm{C}=\mathrm{N}), \quad 135.15, \quad 133.21, \quad 131.13, \quad 127.53,125.10, \quad 125.84(\mathrm{Ar}-\mathrm{C})$, 50.01 $\left(\mathrm{CH}_{2} \mathrm{Cl}\right), 38.75\left(\mathrm{CH}_{2}, \mathrm{CH}_{2} \mathrm{CO}\right), 27.41\left(\mathrm{CH}_{2}\right)$. ESI-MS $(\mathrm{m} / \mathrm{z}): 290\left(\mathrm{M}^{+}\right)$. Anal.calcd. for $\mathrm{C}_{13} \mathrm{H}_{11} \mathrm{ClN}_{4} \mathrm{O}_{2}$ : C, 53.71; H, 3.81; N, 19.27. Found: $\mathrm{C}, 53.62 ; \mathrm{H}$, $3.83 ; \mathrm{N}, 19.41$.

2.3. General procedure for synthesis of 1-(1H-benzo[d]imidazol-2-yl)-3-(5(methyl substituted)-1,3,4-oxadiazol-2-yl)propan-1-one (4a-1)

The suspension of compound $4(0.003 \mathrm{~mol})$ and a secondary amine (equimolar; $0.003 \mathrm{~mol})$ in absolute ethanol $(10 \mathrm{~mL})$ was placed into a microwave reaction vessel equipped with a magnetic stir bar and adding sodium acetate $(0.001 \mathrm{~mol})$ The reaction vessel was placed into the scientific microwave synthesizer (model No. CATA-R, Catalyst systems, India) and irradiated at a power level of $5(50 \%$, $350 \mathrm{~W}$ ) for 8-13 min. The completion of reaction was checked by single-spot TLC by using Toluene: Ethyl acetate: Formic acid (5:4:1) and Benzene: Acetone $(9: 1)$ solvent system. The reaction mixture was cooled, poured onto crushed ice and acidified with glacial acetic acid to yield a solid mass. It was filtered and washed with water to remove the inorganic components, dried and recrystallized with carbinol

2.3.1. Synthesis of 1-(1H-benzo[d]imidazol-2-yl)-3-(5-((diethylamino) methyl)-1,3,4-oxadiazol-2-yl) propan-1-one (4a)

Yield: 78\%; Mp. 242-243 ${ }^{\circ} \mathrm{C} ; \mathrm{R}_{\mathrm{f}}=0.49$ (T:E:F). IR $\left(\mathrm{KBr}, \mathrm{cm}^{-1}\right)$ : 3309(N-H), $3072(\mathrm{C}-\mathrm{H}, \mathrm{Ar}-\mathrm{H}), 2950\left(\mathrm{C}-\mathrm{H}, \mathrm{CH}_{2}\right), 2873\left(\mathrm{C}-\mathrm{H}, \mathrm{CH}_{3}\right), 1715(\mathrm{C}=\mathrm{O}), 1670(\mathrm{C}=\mathrm{N})$, 1547(C=C), 1373(N-N=C), 1184(C-O-C, asymmetric), 1077(C-O-C, symmetric). ${ }^{1} \mathrm{H}-\mathrm{NMR}\left(\mathrm{CDCl}_{3}\right): \delta 12.04\left(\mathrm{~s}, 1 \mathrm{H}, \mathrm{NH}, \mathrm{D}_{2} \mathrm{O}\right.$ exchangeable), $7.73(\mathrm{~d}$, $1 \mathrm{H}, J=7.8 . \mathrm{Hz}, \mathrm{H}-4$, benzimidazole $), 7.42(\mathrm{~d}, 1 \mathrm{H}, J=11.7 \mathrm{~Hz}, \mathrm{H}-7$, benzimidazole), $7.28(\mathrm{t}, 2 \mathrm{H}, J=8.7 \mathrm{~Hz}, J=9.6 \mathrm{~Hz}, \mathrm{H}-5,6$, benzimidazole), $4.13(\mathrm{q}$, $\left.4 \mathrm{H}, J=5.4 \mathrm{~Hz}, 2 \mathrm{xCH}_{2}\right), 3.23\left(\mathrm{t}, 2 \mathrm{H}, J=6.9 \mathrm{~Hz}, \mathrm{CH}_{2}\right), 2.87\left(\mathrm{t}, 2 \mathrm{H}, J=6.6 \mathrm{~Hz}, \mathrm{CH}_{2}\right.$ $\left.\mathrm{CH}_{2} \mathrm{CO}\right), 2.60\left(\mathrm{~s}, 2 \mathrm{H}, \mathrm{CH}_{2}\right), 1.25\left(\mathrm{t}, 6 \mathrm{H}, J=1.2 \mathrm{~Hz}, 2 \mathrm{xCH}_{3}\right) .{ }^{13} \mathrm{C}-\mathrm{NMR}\left(\mathrm{CDCl}_{3}\right): \delta$ $171.50(\mathrm{C}=\mathrm{O}), 165.11,161.19(2 \mathrm{C}$, oxadiazole $), 155.02(\mathrm{C}=\mathrm{N}), 132.83,131.96$, $129.86,128.52,124.73,123.48(\mathrm{Ar}-\mathrm{C}), 60.24\left(\mathrm{CH}_{2}, \mathrm{CH}_{2} \mathrm{~N}\right), 30.21\left(\mathrm{CH}_{2}, \mathrm{CH}_{2} \mathrm{CO}\right)$, 28.08 $\left(\mathrm{CH}_{2}\right), 14.60\left(\mathrm{CH}_{3}\right)$. ESI-MS $(\mathrm{m} / \mathrm{z})$ : $327\left(\mathrm{M}^{+}\right)$. Anal.calcd. for $\mathrm{C}_{17} \mathrm{H}_{21} \mathrm{~N}_{5} \mathrm{O}_{2}$ : C, 62.37; H, 6.47; N, 21.39. Found: C, 63.87; H, 6.51; N, 21.35.

2.3.2. Synthesis of 1-(1H-benzo[d]imidazol-2-yl)-3-(5-((diphenylamino)methyl)1,3,4-oxadiazol-2-yl) propan-1-one (4b)

Yield: $80 \%$; Mp. $243-244^{\circ} \mathrm{C} ; \mathrm{R}_{\mathrm{f}}=0.45(\mathrm{~T}: \mathrm{E}: \mathrm{F}) . \mathrm{IR}\left(\mathrm{KBr}, \mathrm{cm}^{-1}\right): 3290(\mathrm{~N}-\mathrm{H})$, 3067(C-H, Ar-H), 2957(C-H, $\left.\mathrm{CH}_{2}\right), 1690(\mathrm{C}=\mathrm{O}), 1654(\mathrm{C}=\mathrm{N}), 1583(\mathrm{C}=\mathrm{C})$, $1287\left(\mathrm{~N}-\mathrm{N}=\mathrm{C}\right.$ ), 1162 (C-O-C, asymmetric), 1027 (C-O-C, symmetric). ${ }^{1} \mathrm{H}-\mathrm{NMR}$ (DMSO- $\left.d_{6}\right): \delta 12.13\left(\mathrm{~s}, 1 \mathrm{H}, \mathrm{NH}, \mathrm{D}_{2} \mathrm{O}\right.$ exchangeable), $7.92(\mathrm{~d}, 1 \mathrm{H}, J=8.7 \mathrm{~Hz}, \mathrm{H}-$ 4, benzimidazole), $7.70(\mathrm{~d}, 1 \mathrm{H}, J=7.8 \mathrm{~Hz}, \mathrm{H}-7$, benzimidazole $), 7.48(\mathrm{t}, 2 \mathrm{H}, J=$ $7.5 \mathrm{~Hz}, \mathrm{H}-5,6$, benzimidazole), 7.27-6.87(m, $10 \mathrm{H}$, phenyl), 3.72(s, $\left.2 \mathrm{H}, \mathrm{CH}_{2}\right)$ $3.16\left(\mathrm{t}, 2 \mathrm{H}, J=7.2 \mathrm{~Hz}, \mathrm{CH}_{2}\right), 2.54\left(\mathrm{t}, 2 \mathrm{H}, J=7.2 \mathrm{~Hz}, \mathrm{CH}_{2}, \mathrm{CH}_{2} \mathrm{CO}\right) .{ }^{13} \mathrm{C}-\mathrm{NMR}$ $\left(\right.$ DMSO- $\left.d_{6}\right): \delta 173.43(\mathrm{C}=\mathrm{O}), 163.51,162.73(2 \mathrm{C}$, oxadiazole $), 157.23(\mathrm{C}=\mathrm{N})$, $150.43,149.27,148.03,133.17,132.56,128.12,127.53,127.02,124.35,123.43$, 122.73, 120.47 (Ar-C), 57.48 $\left(\mathrm{CH}_{2}, \mathrm{CH}_{2} \mathrm{~N}\right), 33.71\left(\mathrm{CH}_{2}, \mathrm{CH}_{2} \mathrm{CO}\right), 25.32\left(\mathrm{CH}_{2}\right)$. ESI-MS $(m / z): 423\left(\mathrm{M}^{+}\right)$. Anal.calcd. for $\mathrm{C}_{25} \mathrm{H}_{21} \mathrm{~N}_{5} \mathrm{O}_{2}: \mathrm{C}, 70.91 ; \mathrm{H}, 5.00 ; \mathrm{N}, 16.54$ Found: C, 70.93; H, 5.07; N, 16.68

2.3.3. Synthesis of 1-(1H-benzo[d]imidazol-2-yl)-3-(5-((4methylpiperazin-1-yl)methyl)-1,3,4 -oxadiazol-2-yl) propan-1-one (4c)

Yield: $78 \%$; Mp. $225-226^{\circ} \mathrm{C} ; \mathrm{R}_{\mathrm{f}}=0.59(\mathrm{~B}: \mathrm{A})$. IR $\left(\mathrm{KBr}, \mathrm{cm}^{-1}\right): 3317(\mathrm{~N}-\mathrm{H})$, 3015(C-H, Ar-H), 2941(C-H, CH $)_{2}, 2868\left(\mathrm{C}-\mathrm{H}, \mathrm{CH}_{3}\right), 1711(\mathrm{C}=\mathrm{O}), 1645(\mathrm{C}=\mathrm{N})$, 1576(C=C), 1363(N-N=C), 1183(C-O-C, asymmetric), 1057(C-O-C, symmetric). ${ }^{1} \mathrm{H}-\mathrm{NMR}$ (DMSO- $\left.d_{6}\right): \delta 11.45\left(\mathrm{~s}, 1 \mathrm{H}, \mathrm{NH}, \mathrm{D}_{2} \mathrm{O}\right.$ exchangeable), $7.84(\mathrm{~d}, 1 \mathrm{H}, J=7.5 \mathrm{~Hz}, \mathrm{H}-4$, benzimidazole $), 7.54(\mathrm{~d}, 1 \mathrm{H}, J=7.5 \mathrm{~Hz}, \mathrm{H}-7$, benzimidazole), 7.41(t, $2 \mathrm{H}, J=7.8 \mathrm{~Hz}, \mathrm{H}-5,6$, benzimidazole), 3.74(s, $\left.2 \mathrm{H}, \mathrm{CH}_{2}\right)$, $3.59\left(\mathrm{t}, 4 \mathrm{H}, J=7.8 \mathrm{~Hz}, 2 \times \mathrm{xH}_{2}\right.$, piperazine), $3.15\left(\mathrm{t}, 4 \mathrm{H}, J=6.9 \mathrm{~Hz}, 2 \mathrm{xCH}_{2}-\mathrm{NCH}_{3}\right.$, piperazine), $3.01\left(\mathrm{t}, 2 \mathrm{H}, J=7.2 \mathrm{~Hz}, \mathrm{CH}_{2}\right), 2.77\left(\mathrm{t}, 2 \mathrm{H}, J=7.2 \mathrm{~Hz}, \mathrm{CH}_{2}, \mathrm{CH}_{2} \mathrm{CO}\right)$, 
$1.91\left(\mathrm{~s}, 3 \mathrm{H}, \mathrm{CH}_{3}\right) .{ }^{13} \mathrm{C}-\mathrm{NMR}\left(\mathrm{DMSO}-d_{6}\right): \delta 175.31(\mathrm{C}=\mathrm{O}), 165.32,163.84(2 \mathrm{C}$, oxadiazole), $156.13(\mathrm{C}=\mathrm{N}), 131.52,128.25,124.57,123.61,123.04,122.81(\mathrm{Ar}-$ C), 61.23( $\left.\mathrm{CH}_{2}, \mathrm{CH}_{2} \mathrm{~N}\right), 54.27,54.13,51.83,51.09\left(4 \mathrm{C}\right.$, piperazine), $43.71\left(\mathrm{CH}_{3}\right)$, 35.02 $\left(\mathrm{CH}_{2}, \mathrm{CH}_{2} \mathrm{CO}\right), 26.51\left(\mathrm{CH}_{2}\right)$. ESI-MS $(\mathrm{m} / \mathrm{z})$ : $354\left(\mathrm{M}^{+}\right)$. Anal.calcd. for $\mathrm{C}_{18} \mathrm{H}_{22} \mathrm{~N}_{6} \mathrm{O}_{2}$ : C, 61.00; H, 6.26; N, 23.71. Found: $\mathrm{C}, 61.09 ; \mathrm{H}, 6.27 ; \mathrm{N}, 23.83$.

2.3.4. Synthesis of 1-((5-(3-(1H-benzo[d]imidazol-2-yl)-3-oxopropyl)1,3,4-oxadiazol-2-yl) methyl) indoline-2,3-dione (4d)

Yield: $72 \%$; Mp. $240-241^{\circ} \mathrm{C} ; \mathrm{R}_{\mathrm{f}}=0.68(\mathrm{~T}: \mathrm{E}: \mathrm{F}) . \mathrm{IR}\left(\mathrm{KBr}, \mathrm{cm}^{-1}\right): 3293(\mathrm{~N}-\mathrm{H})$, 3041(C-H, Ar-H), 2957(C-H, CH $), 1716(\mathrm{C}=\mathrm{O}), 1687(\mathrm{C}=\mathrm{N}), 1572(\mathrm{C}=\mathrm{C})$, 1338(N-N=C), 1168(C-O-C, asymmetric), 1052 (C-O-C, symmetric). ${ }^{1} \mathrm{H}-\mathrm{NMR}$ (DMSO- $\left.d_{6}\right): \delta 11.95\left(\mathrm{~s}, 1 \mathrm{H}, \mathrm{NH}, \mathrm{D}_{2} \mathrm{O}\right.$ exchangeable), 8.74-7.52 (m, 4H, phenyl), 7.64(d, $1 \mathrm{H}, J=7.5 . \mathrm{Hz}, \mathrm{H}-4$, benzimidazole $), 7.51(\mathrm{~d}, 1 \mathrm{H}, J=7.5 \mathrm{~Hz}, \mathrm{H}-7$, benzimidazole), 7.30 (t, $2 \mathrm{H}, J=7.8 \mathrm{~Hz}, \mathrm{H}-5,6$, benzimidazole), $4.36\left(\mathrm{~s}, 2 \mathrm{H}, \mathrm{CH}_{2}\right)$, $3.07\left(\mathrm{t}, 2 \mathrm{H}, J=7.2 \mathrm{~Hz}, \mathrm{CH}_{2}\right), 2.63\left(\mathrm{t}, 2 \mathrm{H}, J=7.2 \mathrm{~Hz}, \mathrm{CH}_{2}, \mathrm{CH}_{2} \mathrm{CO}\right) .{ }^{13} \mathrm{C}-\mathrm{NMR}$ (DMSO- $\left.d_{6}\right): \delta 177.65\left(\mathrm{C}=\mathrm{O}, \mathrm{COCH}_{2}\right), 175.23(\mathrm{CO}), 170.31(\mathrm{C}=\mathrm{O}, \mathrm{CON}), 161.83$, 159.75(2C, oxadiazole), 154.97(C=N), 147.23, 135.76, 133.63, 132.23, 124.35, $124.02,123.43,122.73,122.16,121.43,120.73(\mathrm{Ar}-\mathrm{C}), 56.89\left(\mathrm{CH}_{2}, \mathrm{CH}_{2} \mathrm{~N}\right)$, 34.85( $\left.\mathrm{CH}_{2}, \mathrm{CH}_{2} \mathrm{CO}\right), \quad 26.53\left(\mathrm{CH}_{2}\right)$. ESI-MS $(\mathrm{m} / \mathrm{z}): \quad 401\left(\mathrm{M}^{+}\right)$. Anal.calcd. for $\mathrm{C}_{21} \mathrm{H}_{15} \mathrm{~N}_{5} \mathrm{O}_{4}$ : C, $62.84 ; \mathrm{H}, 3.77 ; \mathrm{N}, 17.45$. Found: $\mathrm{C}, 62.87 ; \mathrm{H}, 3.73 ; \mathrm{N}, 17.52$.

2.3.5. Synthesis of 3-((5-(3-(1H-benzo[d]imidazol-2-yl)-3-oxopropyl)1,3,4-oxadiazol-2-yl) methyl) pyrimidine-2,4(1H,3H)-dione (4e)

Yield: $86 \%$; Mp. $237-238^{\circ} \mathrm{C} ; \mathrm{R}_{\mathrm{f}}=0.66(\mathrm{~T}: \mathrm{E}: \mathrm{F}) . \mathrm{IR}\left(\mathrm{KBr}, \mathrm{cm}^{-1}\right): 3345(\mathrm{~N}-\mathrm{H})$, 3028(C-H, Ar-H), 2911(C-H, $\left.\mathrm{CH}_{2}\right), 1703(\mathrm{C}=\mathrm{O}), 1681(\mathrm{C}=\mathrm{N}), 1578(\mathrm{C}=\mathrm{C})$, 1316(N-N=C), 1193(C-O-C, asymmetric), 1034 (C-O-C, symmetric). ${ }^{1} \mathrm{H}-\mathrm{NMR}$ (DMSO- $\left.d_{6}\right): \delta 11.00\left(\mathrm{~s}, 1 \mathrm{H}, \mathrm{NH}\right.$, benzimidazole, $\mathrm{D}_{2} \mathrm{O}$ exchang eable), $10.81(\mathrm{~s}$, $1 \mathrm{H}, \mathrm{NH}, \mathrm{D}_{2} \mathrm{O}$ exchangeable), 7.81(d, $1 \mathrm{H}, J=7.8 \mathrm{~Hz}, \mathrm{H}-4$, benzimidazole), 7.54 $(\mathrm{d}, 1 \mathrm{H}, J=7.5 \mathrm{~Hz}, \mathrm{H}-7$, benzimidazole), $7.27(\mathrm{t}, 2 \mathrm{H}, J=7.5 \mathrm{~Hz}, \mathrm{H}-5,6$, benzimidazole), $4.32\left(\mathrm{~s}, 2 \mathrm{H}, \mathrm{CH}_{2}\right), 3.23\left(\mathrm{t}, 2 \mathrm{H}, J=7.2 \mathrm{~Hz}, \mathrm{CH}_{2}\right), 2.57(\mathrm{t}, 2 \mathrm{H}, J=$ $\left.6.9 \mathrm{~Hz}, \mathrm{CH}_{2}, \mathrm{CH}_{2} \mathrm{CO}\right) .{ }^{13} \mathrm{C}-\mathrm{NMR}$ (DMSO- $\left.d_{6}\right): \delta 173.51(\mathrm{C}=\mathrm{O}), 170.38(\mathrm{CO}$, CON $), 159.02(\mathrm{C}=\mathrm{O}, \mathrm{NCON}), 164.75,162.86(2 \mathrm{C}$, oxadiazole $), 156.46(\mathrm{C}=\mathrm{N})$, $132.45,131.64,129.84,124.76,123.43,122.53,121.43,118.83(\mathrm{Ar}-\mathrm{C})$, 58.96 $\left(\mathrm{CH}_{2}, \mathrm{CH}_{2} \mathrm{~N}\right), 38.54\left(\mathrm{CH}_{2}, \mathrm{CH}_{2} \mathrm{CO}\right), 28.57\left(\mathrm{CH}_{2}\right)$. ESI-MS $(\mathrm{m} / \mathrm{z}): 366\left(\mathrm{M}^{+}\right)$. Anal.calcd. for $\mathrm{C}_{17} \mathrm{H}_{14} \mathrm{~N}_{6} \mathrm{O}_{4}$ : C, 55.74; H, 3.85; N, 22.94. Found: C, 55.71; H, $3.85 ; \mathrm{N}, 22.82$

2.3.6. Synthesis of 3-(5-((6-amino-9H-purin-9-yl)methyl)-1,3,4-oxadiazol2-yl)-1-(1H-benzo [d] imidazol-2-yl)propan-1-one (4f)

Yield: $90 \%$; Mp. $225-226^{\circ} \mathrm{C} ; \mathrm{R}_{\mathrm{f}}=0.67(\mathrm{~T}: \mathrm{E}: \mathrm{F}) . \mathrm{IR}\left(\mathrm{KBr}, \mathrm{cm}^{-1}\right): 3352(\mathrm{~N}-\mathrm{H})$, $3027(\mathrm{C}-\mathrm{H}, \mathrm{Ar}-\mathrm{H}), 2987\left(\mathrm{C}-\mathrm{H}, \mathrm{CH}_{2}\right), 1716(\mathrm{C}=\mathrm{O}), 1682(\mathrm{C}=\mathrm{N}), 1568(\mathrm{C}=\mathrm{C})$, 1312(N-N=C), 1160(C-O-C, asymmetric), 1022(C-O-C, symmetric), 834(CN). ${ }^{1} \mathrm{H}-\mathrm{NMR}\left(\mathrm{CDCl}_{3}\right): \delta 12.63\left(\mathrm{~s}, 1 \mathrm{H}, \mathrm{NH}, \mathrm{D}_{2} \mathrm{O}\right.$ exchangeable, benzimidazole), $8.51\left(\mathrm{~s}, 2 \mathrm{H}, \mathrm{NH}_{2}, \mathrm{D}_{2} \mathrm{O}\right.$ exchangeable, adenine $), 8.37(\mathrm{~s}, 1 \mathrm{H}$, adenine $), 7.97(\mathrm{~s}, 1 \mathrm{H}$, adenine), $7.70(\mathrm{~d}, 1 \mathrm{H}, J=7.5 \mathrm{~Hz}, \mathrm{H}-4$, benzimidazole), $7.49(\mathrm{t}, 1 \mathrm{H}, J=7.8 \mathrm{~Hz}, \mathrm{H}-$ 7, benzimidazole), $7.27(\mathrm{t}, 2 \mathrm{H}, J=7.5 \mathrm{~Hz}, \mathrm{H}-5,6$, benzimidazole), $4.17(\mathrm{~s}, 2 \mathrm{H}$, $\mathrm{CH}_{2}$, adenine), 2.96(t, $\left.2 \mathrm{H}, J=7.2 \mathrm{~Hz}, \mathrm{CH}_{2}\right), 2.51\left(\mathrm{t}, 2 \mathrm{H}, J=7.2 \mathrm{~Hz}, \mathrm{CH}_{2}, \mathrm{CH}_{2} \mathrm{CO}\right.$ ). ${ }^{13} \mathrm{C}-\mathrm{NMR} \quad\left(\mathrm{CDCl}_{3}\right): \quad \delta \quad 175.31(\mathrm{C}=\mathrm{O}), \quad 161.74, \quad 160.12(2 \mathrm{C}, \quad$ oxadiazole $)$, $153.17(\mathrm{C}=\mathrm{N}), 141.32,138.61,137.52,136.79,134.92,129.81,129.03,128.56$, 127.63, 124.74, 122.87(Ar-C), 63.41( $\mathrm{CH}_{2}$, adenine), 30.72( $\left.\mathrm{CH}_{2}, \mathrm{CH}_{2} \mathrm{CO}\right)$, 28.45 $\left(\mathrm{CH}_{2}\right)$. ESI-MS $(\mathrm{m} / \mathrm{z}): 389\left(\mathrm{M}^{+}\right)$. Anal. calcd. for $\mathrm{C}_{18} \mathrm{H}_{15} \mathrm{~N}_{9} \mathrm{O}_{2}: \mathrm{C}, 55.52 ; \mathrm{H}$, 3.88; N, 32.38. Found: C, 55.86; H, 3.97; N, 32.47.

2.3.7. Synthesis of 1-((5-(3-(1H-benzo[d]imidazol-2-yl)-3-oxopropyl)1,3,4-oxadiazol-2-yl) methyl)-1 H-purin-6(9H)-one (4g)

Yield: $87 \%$; Mp. $233-234^{\circ} \mathrm{C} ; \mathrm{R}_{\mathrm{f}}=0.65(\mathrm{~T}: \mathrm{E}: \mathrm{F}) . \mathrm{IR}\left(\mathrm{KBr}, \mathrm{cm}^{-1}\right): 3364(\mathrm{~N}-\mathrm{H})$, 3086(C-H, Ar-H), 2952(C-H, $\left.\mathrm{CH}_{2}\right), 1724(\mathrm{C}=\mathrm{O}), 1672(\mathrm{C}=\mathrm{N}), 1480(\mathrm{C}=\mathrm{C})$, 1328(N-N=C), 1116(C-O-C, asymmetric), 1020(C-O-C, symmetric). ${ }^{1} \mathrm{H}-\mathrm{NMR}$ $\left(\mathrm{CDCl}_{3}\right): \delta 12.31\left(\mathrm{~s}, 1 \mathrm{H}, \mathrm{NH}, \mathrm{D}_{2} \mathrm{O}\right.$ exchangeable, benzimidazole), 10.87(s, H, $\mathrm{NH}, \mathrm{D}_{2} \mathrm{O}$ exchangeable, guanine), $8.02(\mathrm{~s}, 1 \mathrm{H}$, guanine), $7.85(\mathrm{~s}, 1 \mathrm{H}$, guanine), $7.78(\mathrm{~d}, 1 \mathrm{H}, J=7.8 \mathrm{~Hz}, \mathrm{H}-4$, benzimidazole $), 7.40(\mathrm{t}, 1 \mathrm{H}, J=7.8 \mathrm{~Hz}, \mathrm{H}-7$, benzimidazole), 7.27(t, $2 \mathrm{H}, J=7.5 \mathrm{~Hz}, \mathrm{H}-5,6$, benzimidazole), $3.92\left(\mathrm{~s}, 2 \mathrm{H}, \mathrm{CH}_{2}\right.$, guanine), $3.23\left(\mathrm{t}, 2 \mathrm{H}, J=6.9 \mathrm{~Hz}, \mathrm{CH}_{2}\right), 2.85\left(\mathrm{t}, 2 \mathrm{H}, J=7.2 \mathrm{~Hz}, \mathrm{CH}_{2}, \mathrm{CH}_{2} \mathrm{CO}\right) .{ }^{13} \mathrm{C}-$ NMR $\left(\mathrm{CDCl}_{3}\right): \delta 168.73(\mathrm{C}=\mathrm{O}), 166.23(\mathrm{C}=\mathrm{O}$, guanine $), 158.45,157.31(2 \mathrm{C}$, oxadiazole $), 156.85(\mathrm{C}=\mathrm{N}), 137.93,137.12,136.87,134.92,127.53,126.74$, $124.56,124.01,122.87,120.91(\mathrm{Ar}-\mathrm{C}), 57.37\left(\mathrm{CH}_{2}\right.$, guanine $)$, 38.61 $\left(\mathrm{CH}_{2}\right.$, $\left.\mathrm{CH}_{2} \mathrm{CO}\right)$, 25.43 $\left(\mathrm{CH}_{2}\right)$. ESI-MS $(\mathrm{m} / \mathrm{z})$ : $390\left(\mathrm{M}^{+}\right)$. Anal.calcd. for $\mathrm{C}_{18} \mathrm{H}_{14} \mathrm{~N}_{8} \mathrm{O}_{3}: \mathrm{C}$, 55.38; H, 3.61; N, 28.71. Found: C, 55.41; H, 3.75; N, 28.92.
2.3.8. Synthesis of 3-(5-((1H-1,2,4-triazol-1-yl)methyl)-1,3,4-oxadiazol-2yl)-1-(1H-benzo [d] imidazol-2-yl)propan-1-one (4h)

Yield: $92 \%$; Mp. $221-222^{\circ} \mathrm{C} ; \mathrm{R}_{\mathrm{f}}=0.55(\mathrm{~T}: \mathrm{E}: \mathrm{F}) . \mathrm{IR}\left(\mathrm{KBr}, \mathrm{cm}^{-1}\right): 3321(\mathrm{~N}-\mathrm{H})$, 3178(C-H, Ar-H), 2819(C-H, $\left.\mathrm{CH}_{2}\right), 1724(\mathrm{C}=\mathrm{O}), 1662(\mathrm{C}=\mathrm{N}), 1523(\mathrm{C}=\mathrm{C})$, 1384(N-N=C), 1191(C-O-C, asymmetric), 1033(C-O-C, symmetric), 848(C-N) ${ }^{1} \mathrm{H}-\mathrm{NMR}\left(\mathrm{CDCl}_{3}\right): \delta 12.03\left(\mathrm{~s}, 1 \mathrm{H}, \mathrm{NH}, \mathrm{D}_{2} \mathrm{O}\right.$ exchangeable), 8.04(s, $1 \mathrm{H}, \mathrm{H}-5$, triazole), 7.88(s, $1 \mathrm{H}, \mathrm{H}-3$, triazole), 7.79(d, $1 \mathrm{H}, J=8.1 \mathrm{~Hz}, \mathrm{H}-4$, benzimidazole), 7.63(t, $1 \mathrm{H}, J=7.2 \mathrm{~Hz}, \mathrm{H}-7$, benzimidazole), 7.45(t, $2 \mathrm{H}, J=7.2 \mathrm{~Hz}, \mathrm{H}-5,6$, benzimidazole), $4.73\left(\mathrm{~s}, 2 \mathrm{H}, \mathrm{CH}_{2}\right.$, triazole), $3.29\left(\mathrm{t}, 2 \mathrm{H}, J=6.9 \mathrm{~Hz}, \mathrm{CH}_{2}\right), 2.87(\mathrm{t}$, $\left.2 \mathrm{H}, J=6.9 \mathrm{~Hz}, \mathrm{CH}_{2}, \mathrm{CH}_{2} \mathrm{CO}\right) \cdot{ }^{13} \mathrm{C}-\mathrm{NMR}\left(\mathrm{CDCl}_{3}\right): \delta 172.87(\mathrm{C}=\mathrm{O}), 165.11$, 161.19(2C, oxadiazole), 155.05(C=N), 132.83, 131.96, 129.86, 128.52, 124.73, 123.48, 117.67, 115.68(Ar-C), 60.24(C, triazole), 30.21 $\left(\mathrm{CH}_{2}, \mathrm{CH}_{2} \mathrm{CO}\right)$, 28.20 $\left(\mathrm{CH}_{2}\right)$. ESI-MS $(\mathrm{m} / \mathrm{z}): 323\left(\mathrm{M}^{+}\right)$. Anal.calcd. for $\mathrm{C}_{15} \mathrm{H}_{13} \mathrm{~N}_{7} \mathrm{O}_{2}: \mathrm{C}, 55.72 ; \mathrm{H}$, 4.05; N, 30.33. Found: C, 55.75; H, 4.13; N, 30.39.

2.3.9. Synthesis of 3-(5-((4H-1,2,4-triazol-4-ylamino)methyl)-1,3,4oxadiazol-2-yl)-1-(1H-benzo[d] imidazol-2-yl)propan-1-one (4i)

Yield: $85 \%$; Mp. $215-217^{\circ} \mathrm{C} ; \mathrm{R}_{\mathrm{f}}=0.53(\mathrm{~B}: \mathrm{A})$. IR $\left(\mathrm{KBr}, \mathrm{cm}^{-1}\right): 3317(\mathrm{~N}-\mathrm{H})$, 3104(C-H, Ar-H), 2994 $\left(\mathrm{CH}_{2}\right), 1698(\mathrm{C}=\mathrm{O}), 1612(\mathrm{C}=\mathrm{N}), 1574(\mathrm{C}=\mathrm{C}), 1356(\mathrm{~N}-$ $\mathrm{N}=\mathrm{C}), 1178\left(\mathrm{C}-\mathrm{O}-\mathrm{C}\right.$, asymmetric), 1036(C-O-C, symmetric), 834(C-N). ${ }^{1} \mathrm{H}-$ NMR $\left(\mathrm{CDCl}_{3}\right): \delta 12.47\left(\mathrm{~s}, 1 \mathrm{H}, \mathrm{NH}, \mathrm{D}_{2} \mathrm{O}\right.$ exchangeable), $7.89(\mathrm{~s}, 2 \mathrm{H}$, triazole), $7.60(\mathrm{~d}, 1 \mathrm{H}, J=7.8 \mathrm{~Hz}, \mathrm{H}-4$, benzimidazole), $7.33(\mathrm{t}, 1 \mathrm{H}, J=7.5 \mathrm{~Hz}, \mathrm{H}-7$, benzimidazole), $7.26\left(\mathrm{t}, 2 \mathrm{H}, J=7.5 \mathrm{~Hz}, \mathrm{H}-5,6\right.$, benzimidazole), $3.72\left(\mathrm{~s}, 2 \mathrm{H}, \mathrm{CH}_{2}\right.$ $\mathrm{CH}_{2} \mathrm{NH}$ ), 3.21(t, $\left.2 \mathrm{H}, J=6.9 \mathrm{~Hz}, \mathrm{CH}_{2}\right), 2.92\left(\mathrm{t}, 2 \mathrm{H}, J=6.9 \mathrm{~Hz}, \mathrm{CH}_{2}, \mathrm{CH}_{2} \mathrm{CO}\right.$ ), $2.63(\mathrm{~s}, \mathrm{IH}, \mathrm{NH}) .{ }^{13} \mathrm{C}-\mathrm{NMR}\left(\mathrm{CDCl}_{3}\right): \delta 176.10(\mathrm{C}=\mathrm{O}), 163.41,160.85(2 \mathrm{C}$ oxadiazole), 155.71( $\mathrm{C}=\mathrm{N}), 137.32(\mathrm{C}$, aminotriazole), 129.63, 128.91, 124.73, 123.86, 121.18, 120.83(Ar-C), 66.51 $\left(\mathrm{CH}_{2}, \mathrm{NHCH}_{2}\right), 32.68\left(\mathrm{CH}_{2}, \mathrm{CH}_{2} \mathrm{CO}\right)$, 28.36 $\left(\mathrm{CH}_{2}\right)$. ESI-MS $(\mathrm{m} / \mathrm{z})$ : $338\left(\mathrm{M}^{+}\right)$. Anal.calcd. for $\mathrm{C}_{15} \mathrm{H}_{14} \mathrm{~N}_{8} \mathrm{O}_{2}: \mathrm{C}, 53.25 ; \mathrm{H}$, $4.17 ; \mathrm{N}, 33.12$. Found: $\mathrm{C}, 53.43 ; \mathrm{H}, 4.25 ; \mathrm{N}, 33.71$.

2.3.10. Synthesis of 3-(5-((1H-imidazol-1-yl)methyl)-1,3,4-oxadiazol-2-yl)1-(1H-benzo[d] imidazol-2-yl) propan-1-one $(4 \mathrm{j})$

Yield: $82 \%$; Mp. $207-208^{\circ} \mathrm{C} ; \mathrm{R}_{\mathrm{f}}=0.57(\mathrm{~T}: \mathrm{E}: \mathrm{F}) . \mathrm{IR}\left(\mathrm{KBr}, \mathrm{cm}^{-1}\right): 3348(\mathrm{~N}-\mathrm{H})$ $3054(\mathrm{C}-\mathrm{H}, \mathrm{Ar}-\mathrm{H}), 2942\left(\mathrm{C}-\mathrm{H}, \mathrm{CH}_{2}\right), 1726(\mathrm{C}=\mathrm{O}), 1685(\mathrm{C}=\mathrm{N}), 1532(\mathrm{C}=\mathrm{C})$, $1328(\mathrm{~N}-\mathrm{N}=\mathrm{C}), 1176$ (C-O-C, asymmetric), 1042 (C-O-C, symmetric). ${ }^{1} \mathrm{H}-\mathrm{NMR}$ $\left(\mathrm{CDCl}_{3}\right): \delta 11.87\left(\mathrm{~s}, 1 \mathrm{H}, \mathrm{NH}, \mathrm{D}_{2} \mathrm{O}\right.$ exchangeable), 8.07 (s, $1 \mathrm{H}, \mathrm{H}-2$, imidazole), $7.74(\mathrm{~d}, 1 \mathrm{H}, J=7.5 \mathrm{~Hz}, \mathrm{H}-4$, benzimidazole), $7.30(\mathrm{t}, 1 \mathrm{H}, J=7.5 \mathrm{~Hz}, \mathrm{H}-7$, benzimidazole), 7.27(t, $2 \mathrm{H}, J=7.5 \mathrm{~Hz}, \mathrm{H}-5,6$, benzimidazole), $6.65(\mathrm{~d}, 1 \mathrm{H}, J=$ $7.2 \mathrm{~Hz}, \mathrm{H}-5$, imidazole), $6.48\left(\mathrm{~d}, 1 \mathrm{H}, J=8.1 \mathrm{~Hz}, \mathrm{H}-4\right.$, imidazole), $4.03\left(\mathrm{~s}, 2 \mathrm{H}, \mathrm{CH}_{2}\right.$ imidazole), 3.12(t, $\left.2 \mathrm{H}, J=7.2 \mathrm{~Hz}, \mathrm{CH}_{2}\right), 2.85\left(\mathrm{t}, 2 \mathrm{H}, J=6.9 \mathrm{~Hz}, \mathrm{CH}_{2}, \mathrm{CH}_{2} \mathrm{CO}\right)$ ${ }^{13} \mathrm{C}-\mathrm{NMR} \quad\left(\mathrm{CDCl}_{3}\right): \quad \delta \quad 175.21(\mathrm{C}=\mathrm{O}), \quad 161.92, \quad 158.32(2 \mathrm{C}, \quad$ oxadiazole $)$, 154.63(C=N), 136.25, 132.53, 131.54(3C, imidazole), 126.43, 125.82, 123.35, 122.61, 121.57, 119.64(Ar-C), 60.73( $\mathrm{CH}_{2}$, imidazole), 35.31 $\left(\mathrm{CH}_{2}, \mathrm{CH}_{2} \mathrm{CO}\right)$, $27.93\left(\mathrm{CH}_{2}\right)$. ESI-MS $(\mathrm{m} / z)$ : 322(M $\left.\mathrm{M}^{+}\right)$. Anal.calcd. for $\mathrm{C}_{16} \mathrm{H}_{14} \mathrm{~N}_{6} \mathrm{O}_{2}: \mathrm{C}, 59.62 ; \mathrm{H}$, 4.38; N, 26.07. Found: C, 59.74; H, 4.25; N, 26.35 .

2.3.11. Synthesis of 3-((5-(3-(1H-benzo[d]imidazol-2-yl) -3-oxopropyl)-1, 3, 4-oxadiazol-2-yl) methyl)-5-methylpyrimidine-2,4(1H, 3H)-dione (4k)

Yield: $85 \%$; Mp. $236^{\circ} \mathrm{C} ; \mathrm{R}_{\mathrm{f}}=0.58(\mathrm{~T}: \mathrm{E}: \mathrm{F}) . \mathrm{IR}\left(\mathrm{KBr}, \mathrm{cm}^{-1}\right): 3344(\mathrm{~N}-\mathrm{H})$, 3105(C-H, Ar-H), $2974\left(\mathrm{C}-\mathrm{H}, \mathrm{CH}_{2}\right), 1724(\mathrm{C}=\mathrm{O}), 1662(\mathrm{C}=\mathrm{N}), 1566(\mathrm{C}=\mathrm{C})$ 1384(N-N=C), 1180(C-O-C, asymmetric), 1064(C-O-C, symmetric). ${ }^{1} \mathrm{H}-\mathrm{NMR}$ $\left(\mathrm{CDCl}_{3}\right): \delta 14.10\left(\mathrm{~s}, 1 \mathrm{H}, \mathrm{NH}, \mathrm{D}_{2} \mathrm{O}\right.$ exchangeable, pyrimidine), $12.37(\mathrm{~s}, 1 \mathrm{H}, \mathrm{NH}$, $\mathrm{D}_{2} \mathrm{O}$ exchangeable, benzimidazole $), 8.03(\mathrm{~s}, 1 \mathrm{H}$, pyrimidine $), 7.67(\mathrm{~d}, 1 \mathrm{H}, J=$ $7.8 \mathrm{~Hz}, \mathrm{H}-4$, benzimidazole), $7.43(\mathrm{t}, 1 \mathrm{H}, J=7.2 \mathrm{~Hz}, \mathrm{H}-7$, benzimidazole), $7.29(\mathrm{dd}, 2 \mathrm{H}, J=7.8 \mathrm{~Hz}, J=7.5 \mathrm{~Hz}, \mathrm{H}-5,6$, benzimidazole $), 4.09(\mathrm{~s}, 2 \mathrm{H}$, $\mathrm{CH}_{2}$,pyrimidine), $3.49\left(\mathrm{t}, 2 \mathrm{H}, J=6.9 \mathrm{~Hz}, \mathrm{CH}_{2}\right), 3.09\left(\mathrm{t}, 2 \mathrm{H}, J=6.9 \mathrm{~Hz}, \mathrm{CH}_{2}\right.$, $\left.\mathrm{CH}_{2} \mathrm{CO}\right), 2.57\left(\mathrm{~s}, 3 \mathrm{H}, \mathrm{CH}_{3}\right) .{ }^{13} \mathrm{C}-\mathrm{NMR}\left(\mathrm{CDCl}_{3}\right): \delta 173.61(\mathrm{C}=\mathrm{O}), 173.14$ $172.15(\mathrm{C}=\mathrm{O}$, pyrimidine $), 159.19,159.07(2 \mathrm{C}$, oxadiazole $), 156.42(\mathrm{C}=\mathrm{N})$, $132.64,130.84,129.88,129.84,128.87,128.48,124.16,115.70(\mathrm{Ar}-\mathrm{C})$, 60.52 $\left(\mathrm{CH}_{2}\right.$, pyrimidine), $30.30\left(\mathrm{CH}_{2}, \mathrm{CH}_{2} \mathrm{CO}\right), 28.02\left(\mathrm{CH}_{2}\right), 14.31\left(\mathrm{CH}_{3}\right)$. ESI-MS $(\mathrm{m} / \mathrm{z}): 380\left(\mathrm{M}^{+}\right)$. Anal.calcd. for $\mathrm{C}_{18} \mathrm{H}_{16} \mathrm{~N}_{6} \mathrm{O}_{4}: \mathrm{C}, 56.84 ; \mathrm{H}, 4.24 ; \mathrm{N}, 22.10$. Found: C, 56.87; H, 4.33; N, 22.17

2.3.12. Synthesis of 1-((5-(3-(1H-benzo[d]imidazol-2-yl)-3-oxopropyl)1,3,4-oxadiazol-2-yl) methyl)-4-aminopyrimidin-2(1H)-one (4l)

Yield: $82 \%$; Mp. $234-235^{\circ} \mathrm{C} ; \mathrm{R}_{\mathrm{f}}=0.59$ (T:E:F). IR $\left(\mathrm{KBr}, \mathrm{cm}^{-1}\right): 3372(\mathrm{~N}-\mathrm{H})$, 3043(C-H, Ar-H), 2957(C-H, $\left.\mathrm{CH}_{2}\right), 1703(\mathrm{C}=\mathrm{O}), 1674(\mathrm{C}=\mathrm{N}), 1587(\mathrm{C}=\mathrm{C}), 1372$ 
(N-N=C), 1184(C-O-C, asymmetric), 1065(C-O-C, symmetric). ${ }^{1} \mathrm{H}-\mathrm{NMR}$ $\left(\mathrm{CDCl}_{3}\right): \delta 12.17\left(\mathrm{~s}, 1 \mathrm{H}, \mathrm{NH}, \mathrm{D}_{2} \mathrm{O}\right.$ exchangeable $), \quad 8.34(\mathrm{~d}, 1 \mathrm{H}, J=8.1 \mathrm{~Hz}$, pyrimidine), $8.01\left(\mathrm{~s}, 2 \mathrm{H}, \mathrm{NH}_{2}, \mathrm{D}_{2} \mathrm{O}\right.$ exchangeable), $7.71(\mathrm{~d}, 1 \mathrm{H}, J=7.5 \mathrm{~Hz}, \mathrm{H}-4$, benzimidazole), $7.33(\mathrm{t}, 1 \mathrm{H}, J=7.2 \mathrm{~Hz}, \mathrm{H}-7$, benzimidazole), $7.24(\mathrm{t}, 2 \mathrm{H}, J=$ $8.1 \mathrm{~Hz}, \mathrm{H}-5,6$, benzimidazole), $6.72(\mathrm{~d}, 1 \mathrm{H}, J=7.5 \mathrm{~Hz}$, pyrimidine $), 4.13(\mathrm{~s}, 2 \mathrm{H}$, $\mathrm{CH}_{2}$, pyrimidine), $3.27\left(\mathrm{t}, 2 \mathrm{H}, J=7.2 \mathrm{~Hz}, \mathrm{CH}_{2}\right), 2.76\left(\mathrm{t}, 2 \mathrm{H}, J=6.9 \mathrm{~Hz}, \mathrm{CH}_{2}\right.$, $\left.\mathrm{CH}_{2} \mathrm{CO}\right) .{ }^{13} \mathrm{C}$-NMR $\left(\mathrm{CDCl}_{3}\right): \delta 174.53(\mathrm{C}=\mathrm{O}), 165.01(\mathrm{CO}$, pyrimidine $), 160.23$, 159.10(2C, oxadiazole), $155.41(\mathrm{C}=\mathrm{N}), 138.10,135.29,130.13,129.69,128.43$, 123.14, 120.34, 118.72, 110.51(Ar-C), $55.72\left(\mathrm{CH}_{2}\right.$, pyrimidine), $32.56\left(\mathrm{CH}_{2}\right.$, $\left.\mathrm{CH}_{2} \mathrm{CO}\right)$, 27.13 $\left(\mathrm{CH}_{2}\right)$. ESI-MS $(\mathrm{m} / \mathrm{z}): 365\left(\mathrm{M}^{+}\right)$. Anal.calcd. for $\mathrm{C}_{17} \mathrm{H}_{15} \mathrm{~N}_{7} \mathrm{O}_{3}: \mathrm{C}$, 55.89; H, 4.14; N, 26.84. Found: C, 55.97; H, 3.65; N, 26.95.

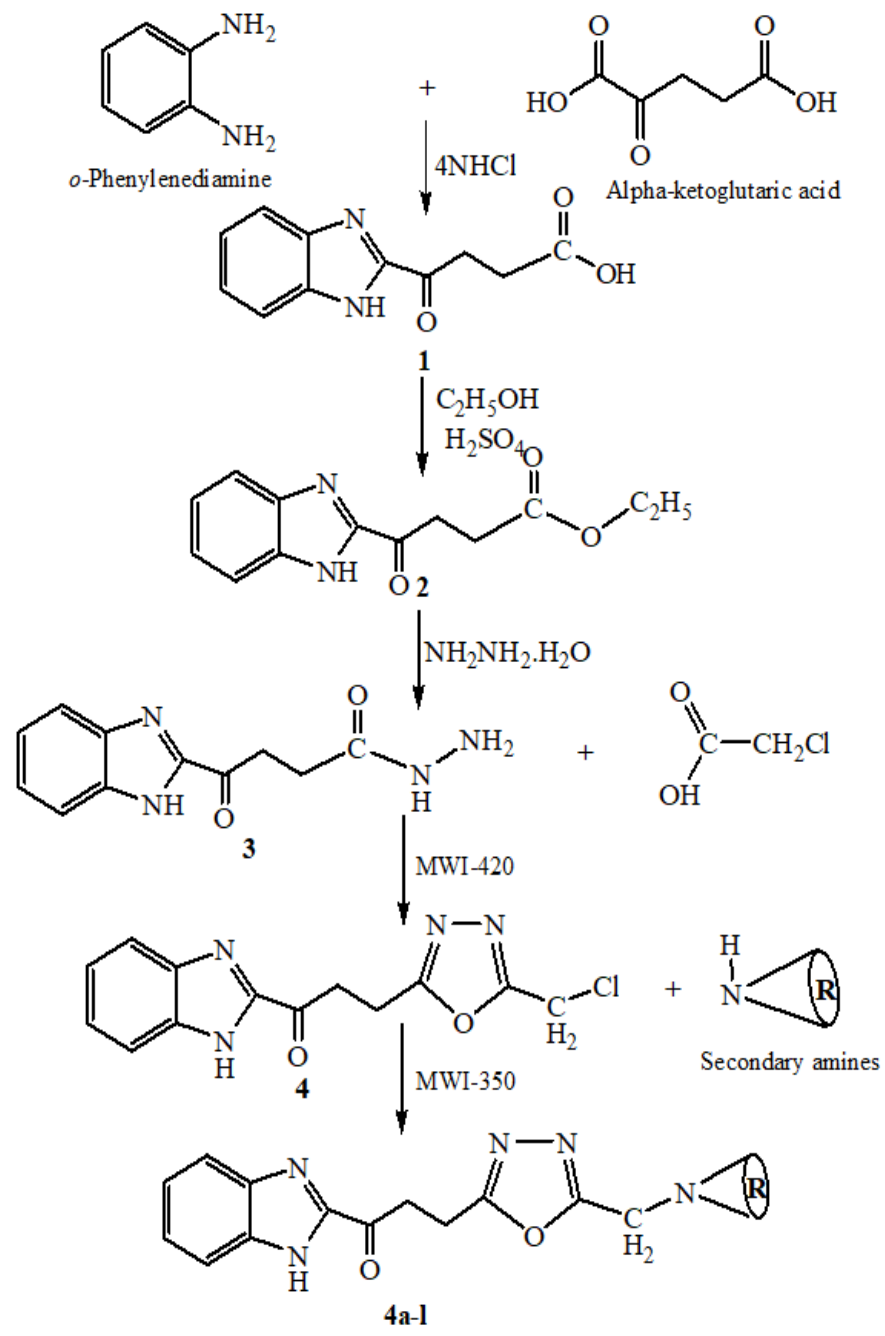

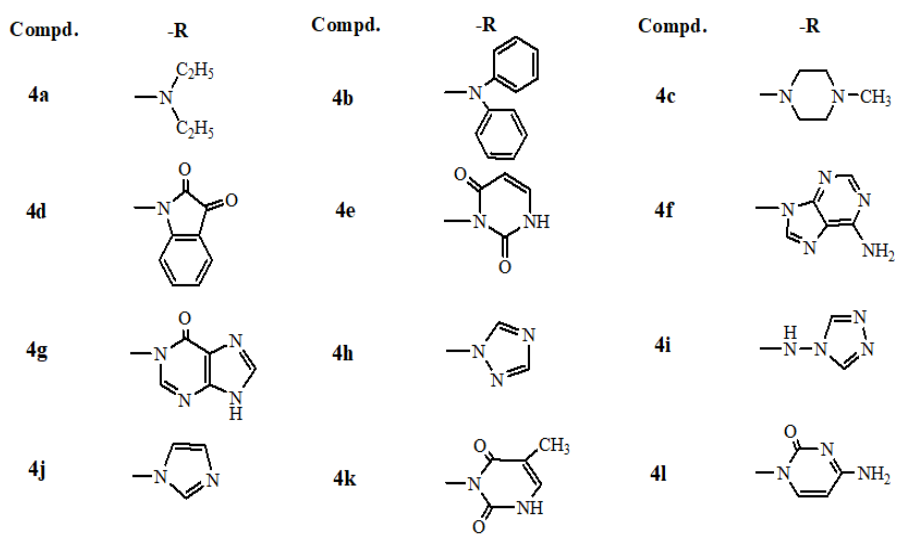

Scheme 1. Synthesis of benzimidazole bearing oxadiazole derivatives (4a-I).

\subsection{In-Silico Analysis Study}

\subsubsection{Drug likeness and Lipinski's rule}

Christopher A. Lipinski was formulated a rule in 1997 of molecular properties for the prediction of drug's pharmacokinetics (ADME; Absorption, distribution, metabolism and excretion) in human body. The modification in molecular structure often leads to drugs with higher molecular weight, more rings, more rotatable bond and a higher lipophilicity. The rule is significant for drug development where a pharmacologically active lead compound is boosted stepwise for increased activity and selectivity as well as drug like properties. The rule states that poor absorption or permeation are more possible when a ligand molecule violates Lipinski rule of 5 , that is has more than five hydrogen bond donors, molecular weight is over $500, \log \mathrm{P}$ is over 5 and sum of $\mathrm{N}$ and $\mathrm{O}$ is over 10 [33]. Drug likeness may be defined as a complex balance of numerous molecular properties and structure features which determine whether particular molecule is similar to the known drugs. These properties, mainly are hydrophobicity, electronic distribution, hydrogen bonding characteristics, molecule size, flexibility and presence of several pharmacophoric features influence the behavior of molecule in a living organism, including bioavailability, transport properties, affinity to proteins, reactivity, toxicity and metabolic stability [34-36].

\subsubsection{Drug-likeness Prediction}

This screening methodology was implemented to analyze the drug likeness of proposed ligands as it influences the behavior of molecule in a living organism, including bioavailability, transport properties, affinity to proteins, reactivity, toxicity and metabolic stability [35-36]. Pre ADMET contains drug-likeness prediction module based on Lipinski's rule. Also, it is possible to use several drug-like rules that defined drug-like characteristics of drug such as Ghose filter [37], CMC [38], WDI [39] and MDDR DB [40]. Ghose filter defines druglikeness constraints as follows: calculated $\log \mathrm{P}$ is between -0.4 and 5.6, molecular weight between 160 and 480, molar refractivity between 40 and 130, and the total number of atoms between 20 and 70 [38]. The filter has been developed that discriminates between drug-like and nondrug-like chemical matter.

\subsubsection{Molinspiration and Prediction of Drug likeness}

Chemical structures and smiles notations of the synthetic compounds were obtained by Chem. Bio draw Ultra software (11.0 versions) (Table 2). Smiles notations of benzimidazole derivatives then fed in Molinspiration (2018.02 version) and drug likeness tool to calculate many molecular properties (Table 3 $\& 4)$ and to predict bioactivity score for drug targets including enzymes and nuclear receptors, kinase inhibitors, GPCR ligands and ion channel modulators (Table 5). Molecular properties such as partition coefficient (Log P), topological polar surface area (TPSA), hydrogen bond donors and acceptors, rotatable bonds, number of atoms, molecular weight and violations of Lipinski's rule of five were calculated to evaluate the drug likeness of the synthesized compounds and represented in Table 3. The absorption percentage ( $\% \mathrm{Ab})$ was also calculated by using the following formula: $\% \mathrm{Ab}=109-[0.345 \times$ TPSA] (Table 3). These properties are calculated on the basis of Lipinski's rule of five, which states that any compound considered as drug should have partition coefficient less than 5, its polar surface area within $140 \AA^{2}$, it should have $\mathrm{H}$ bond acceptor less than 10 , it should have $\mathrm{H}$ bond donor less than 5 and its molecular weight within 500 dalton [41-46]. 
Table 2: Chemical structure and smiles of benzimidazole hybrid with oxadiazole ring (4a-1).

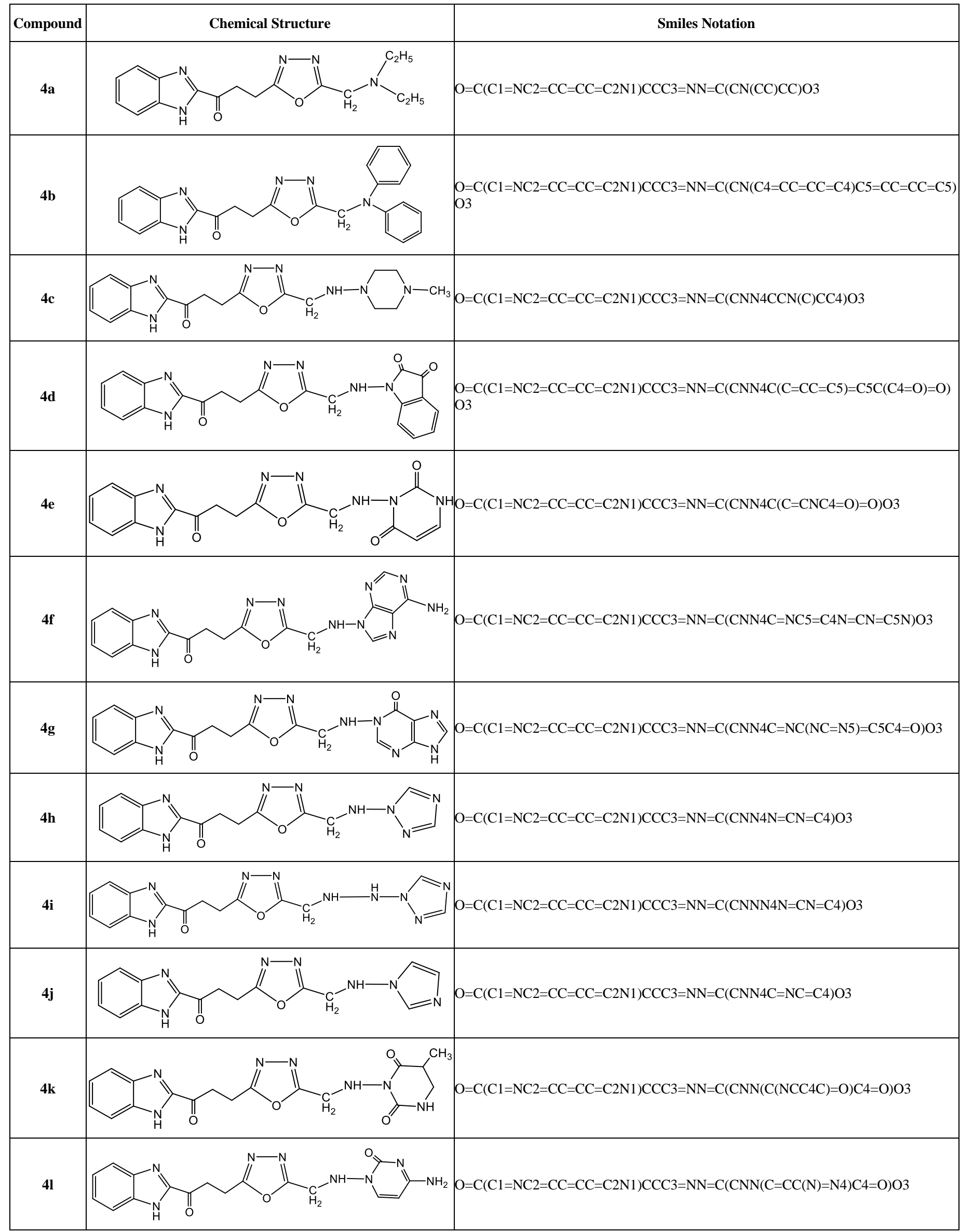


These are following molecular properties which are calculated with the help of Molinspiration. LogP (Octanol/water partition coefficient): The sum of fragment-based contributions and correction factors. TPSA (Topological Polar Surface Area): A sum of fragment contributions. $\mathrm{O}^{-}$and $\mathrm{N}^{-}$centered polar fragments are considered. TPSA has been shown to be a very good descriptor characterizing drug absorption, including intestinal absorption, bioavailability, Caco-2 permeability and blood-brain barrier penetration. Molecular volume: The sum of fragment contributions to real 3D volume for a training set of about twelve thousand, mostly drug-like molecules. Rule of 5 Properties: The rule states, that most drug-like molecules have $\log \mathrm{P}<=5$, molecular weight $<=500$, number of hydrogen bond acceptors $<=10$ and number of hydrogen bond donors $<=5$. Molecules violating more than one of these rules may have problems with bioavailability. The rule is called 'Rule of 5', because the border values are 5, $500,2 * 5$, and 5. Number of Rotatable Bonds (n-rotb): Rotatable bond is defined as any single non-ring bond, bounded to nonterminal heavy (non-hydrogen) atom and a measure of molecular flexibility. Amide $\mathrm{C}-\mathrm{N}$ bonds are not considered because of their high rotational energy barrier [34, 47-51]. Drug likeness: Drug likeness may be defined as a multifarious balance of numerous molecular properties and structure features which determine whether particular molecule is similar to the known drugs. These properties, mainly hydrophobicity, electronic distribution, hydrogen bonding characteristics, molecule size, flexibility and presence of various pharmacophoric features influence the behavior of molecule in a living organism, including bioavailability, transport properties, affinity to proteins, reactivity, toxicity, metabolic stability and many others [33-34, 49-51].

\subsubsection{Osiris Property Explorer and Drug Relevant Properties Prediction}

Property explorer is a free tool to predict physicochemical and toxicological molecular properties, which need to be optimized when designing pharmaceutically active compounds. It was originally developed by T. Sander and used at Actelion Pharmaceutical Ltd. as integrated component of the compound registration system in the drug discovery department. Now days, it is a de-facto standard for physicochemical property prediction and toxicity risk indication. For predicting drug relevant properties of a chemical compound just draw its structure and property explorer will start calculating properties as soon as a chemical structure is valid. Prediction results are valued and color coded [5253].

Properties with high risks of undesired effects like mutagenicity or a poor intestinal absorption are shown in red. Whereas a green color indicates drug conform behavior. Charges should be balanced and atom valances not exceeded. Nitro-groups, for instance, should be drawn with a positive charge on nitrogen and a negative on one of the oxygens with a single bond connecting these two atoms. The drug relevant properties and toxicity risk assessment by OSIRIS property explorer are represented in Table $6 \& 7$. These are the following drug relevant properties and toxicity risk. Molecular weight: Compounds with higher weights are less likely to be absorbed and therefore to ever reach the place of action. lopP prediction: Low hydrophilicities and therefore high $\log \mathrm{P}$ values cause poor absorption or permeation. It has been shown for compounds to have a reasonable probability of being well absorb their $\log \mathrm{P}$ value must not be greater than 5.0. Aqueous Solubility: The aqueous solubility of a compound significantly affects its absorption and distribution characteristics. It shows that more than $80 \%$ of the drugs on the market have a calculated $\log S$ value greater than -4 . Topological polar surface area: The contributions of all polar atoms being exposed to the molecule's surface sum up to an area beyond 80 or $100 \AA^{2}$, then the molecules likelihood to pass membranes easily is significantly reduced. Drug likeness: There are many approaches around that assess a compound's drug likeness partially based on topological descriptors, fingerprints of MDL structure keys or other properties as cLogP and molecular weights. Overall drug score: The drug score combines drug likeness, cLogP, $\log \mathrm{S}$, molecular weight and toxicity risks in one handy value than may be used to judge the compound's overall potential to qualify for a drug [54-60].

\subsubsection{Toxicity Assessment}

While drawing a structure the toxicity risk predictor will start looking for potential toxicity risks as long as the currently drawn structure is a valid chemical entity. Toxicity risk alerts are an indication that the drawn structure may be harmful concerning the risk category specified. However, risk alerts are by no means meant to be a fully reliable toxicity prediction. Prediction results are evaluated and color coded. Unfavorable properties or those with a high risk of side effects like mutagenicity or poor intestinal absorption are shown in red. Green color instead indicates drug conform behavior. The toxicity risk assessment is mandatory to avoid destructive substances for further processing of drug discovery and development. The mutagenic, tumorigenic, irritant and reproductive toxicity risks were measured by means of pre-computed set of structural fragment which was created based on the classification of compounds from the Registry of Toxic Effects of Chemical Substances (RTECS) database. The toxicity risks are estimated with color code. The undesired (toxic risks) effects of molecule are displayed in red and while the green color indicates the desired effects of compound [56-60, 61-62].

\subsubsection{Pre ADMET}

Over $50 \%$ of the candidates failed due to ADME/Toxicity deficiencies during development. To avoid this failure at the development a set of in-vitro ADME/Toxicity screens has been implemented in most pharmaceutical companies with the aim of discarding compounds in the discovery phase that are likely to fail further down the line. Even though the early stage in-vitro ADME reduces the probability of failure at the development stage, it is still time consuming and resource intensive. Pre ADMET consists of main four parts as following. Molecular descriptor calculation: The ADME/Toxicity properties are closely related to physicochemical descriptors such as lipophilicity $(\log \mathrm{P})$ molecular weight, polar surface area and water solubility. Drug likeness prediction: Pre ADMET contains drug-likeness prediction module based on Lipinski's and Lead-like. ADME Prediction: Caco2 cell model and MDCK (Madin darby canine kidney) cell model has been recommended as a reliable invitro model for the prediction of oral drug absorption. Additionally, in-silico HIA (human intestinal absorption) model and skin permeability model can predict and identify potential drug for oral delivery and transdermal delivery. In distribution, BBB (blood brain barrier) penetration can give information of therapeutic drug in the central nervous system (CNS), plasma protein binding model in its disposition and efficacy [37, 39, 62-63].

\subsubsection{Acute toxicity prediction}

Toxicity prediction is the more and more importance in early drug discovery since $30 \%$ of drug candidates fail owing to these issues. The toxicity prediction was done by mutagenicity of Ames test and T.E.S.T. tool (Version 4.2.1) a program from the US Environmental Protection Agency.

Ames test: Ames test is a simple method to test mutagenicity of a compound, which is suggested by Dr. Ames. It uses several strains of bacterium salmonella typhimurium that carry mutations in genes involved in histidine synthesis, so that they require histidine for growth. Ames TA100 (+S9); In-vitro Ames test result in TA100 strain (Metabolic activation by rat liver homogenate). Ames TA100 (S9); In-vitro Ames test result in TA100 strain (No metabolic activation). Ames TA1535 (+S9); In-vitro Ames test result in TA1535 strain (Metabolic activation by rat liver homogenate). Ames TA1535 (-S9); In-vitro Ames test result in TA1535 strain (No metabolic activation) [64-66]. T.E.S.T program tool: This is a software program developed by US environmental protection agency for the assessment of acute toxicity of compound. QSAR/QSPR (Quantitative structureactivity relationship/Quantitative structure-property relationship) descriptor was used in this tool for the prediction of toxicity of synthetic as well as natural compounds. A types of descriptor, which are used in the tool is based on the molecular structures of compound [67-69].

\subsection{Pharmacological evaluation}

All the selected compounds were submitted to National Cancer Institute (NCI), USA for evaluation of their in-vitro anticancer activity at dose $\left(1 \times 10^{-5} \mathrm{M}\right)$ against full NCI 60 cell lines panels representing on full nine human systems as leukemia, melanoma and cancers of lung, colon, brain, breast, ovary, kidney and prostate. The screening is a two-stage process, beginning with the evaluation of all compounds against the 60 cell lines at a single dose. The compounds added at a concentration $\left(1 \times 10^{-5} \mathrm{M}\right)$ and the culture incubated for $48 \mathrm{~h}$. End point determinations made with a protein binding dye, Sulforhodamine B [70-71] Results for each compound were reported as a mean graph of the percent growth of the treated cells. Results of each test agents are reported as percentage growth of the treated cells when compared with untreated control cells [72-73]. The output from the single dose screen is analyzed by the COMPARE program and compounds which exhibit significant growth inhibition at a single dose of $10 \mathrm{mM}$ are further evaluated against the 60 cell panel found results are represented in Table 11 


\section{RESULT AND DISCUSSION}

\subsection{Chemistry}

The purity of compounds was checked by single-spot TLC using Toluene: Ethyl acetate: Formic acid (5:4:1) and Benzene: Acetone (9:1) solvent systems and spots located under iodine vapors/UV light. The structures of the synthesized compounds were established on the basis of modern analytical techniques; IR, ${ }^{1} \mathrm{H}$-NMR, ${ }^{13} \mathrm{C}-\mathrm{NMR}$, Mass spectral data and elemental analysis. The title compounds were synthesized in four steps, as outlined in Scheme 1.

In the first step, the starting material, 4-(1H-benzo[ $d]$ imidazol-2-yl)-4oxobutanoic acid (1), was synthesized by reacting $o$-phenylenediamine with $\alpha$ ketoglutaric acid in presence of $4 \mathrm{NHCl}$. It gave effervescence with sodium bicarbonate solution and showed sharp melting point. It was further identified on the basis of IR, ${ }^{1} \mathrm{H}-\mathrm{NMR},{ }^{13} \mathrm{C}-\mathrm{NMR}$ and massspectral data. The IR spectra of the compound showed peaks at 3394 and $3326 \mathrm{~cm}^{-1}$ for $\mathrm{O}-\mathrm{H}$ and $\mathrm{N}-\mathrm{H}$, respectively. In ${ }^{1} \mathrm{H}-\mathrm{NMR}$ spectra, there appeared a singlet at $\delta 12.97$ indicative of ring $\mathrm{O}-\mathrm{H}$ and another singlet at $\delta 12.34$ for $\mathrm{N}-\mathrm{H}$, both disappeared by the addition of $\mathrm{D}_{2} \mathrm{O}$ as confirmation for these groups. The appeared peak in IR spectra at around1728 and $1600 \mathrm{~cm}^{-1}$ accounted for $\mathrm{C}=\mathrm{O}$ and $\mathrm{C}=\mathrm{N}$. The chemical shift in ${ }^{13} \mathrm{C}-\mathrm{NMR}$ spectra at $\delta 175.5$ and 159.6 could be accounted for $\mathrm{C}=\mathrm{O}$ and $\mathrm{C}=\mathrm{N}$. The ${ }^{1} \mathrm{H}-\mathrm{NMR}$ spectra indicated the two triplets at around $\delta 3.05(\mathrm{~J}=6.9 \mathrm{~Hz})$ and $2.76(\mathrm{~J}=$ $6.9 \mathrm{~Hz}$ ) and in ${ }^{13} \mathrm{C}-\mathrm{NMR}$ appeared peak at $\delta 31.36$ and 34.23 could be accounted for $\mathrm{CH}_{2}$ and $\mathrm{CH}_{2} \mathrm{CO}$, respectively. The presence of two doublet, one triplet in ${ }^{1} \mathrm{H}-$ NMR spectra at around $\delta 7.6(J=7.5 \mathrm{~Hz}), 7.4(J=7.2 \mathrm{~Hz})$ and $7.2(J=8.7 \mathrm{~Hz})$ indicative of benzimidazole hydrogen and ${ }^{13} \mathrm{C}$-NMR appeared peaks at $\delta 138.27$, $132.21,130.19,128.57,124.65$, and 123.64 indicated benzimidazole carbon. The mass spectrum exhibited molecular ion peak at $\mathrm{m} / \mathrm{z} 218$ according to their molecular formula $\mathrm{C}_{11} \mathrm{H}_{10} \mathrm{~N}_{2} \mathrm{O}_{3}$.

In second step, compound $\mathbf{1}$ was treated with hydrazine hydrate through an ester intermediate (2) to furnish 4-(1H-benzo[d]imidazol-2-yl)-4oxobutanehydrazide (3). It showed different TLC spot, $\mathrm{R}_{\mathrm{f}}$ value and melting point while comparing with starting compound 1.It did not react with sodium bicarbonate solution. The IR spectra of compound $\mathbf{3}$ showed characteristic band at 3494 and $3359 \mathrm{~cm}^{-1}$ due to $\mathrm{N}-\mathrm{H}_{2}$ and $\mathrm{N}-H$ group. In ${ }^{1} \mathrm{H}-\mathrm{NMR}$ spectra, there appeared a singlet at $\delta 11.5$ indicative of ring $\mathrm{N}-H$, singlet at $\delta 10.7$ for hydrazide $\mathrm{N}-H$ and another broad singlet at $\delta 9.0$ for $\mathrm{NH}_{2}$ group, three disappeared by addition of $\mathrm{D}_{2} \mathrm{O}$ as confirmation for these groups. The appeared peak in IR spectra at around 1691 and $1643 \mathrm{~cm}^{-1}$ accounted for $\mathrm{C}=\mathrm{O}$ and $\mathrm{C}=\mathrm{N}$. The chemical shift in ${ }^{13} \mathrm{C}$-NMR spectra at $\delta 179.1$ and 156.3 could be accounted for $\mathrm{C}=\mathrm{O}$ and $\mathrm{C}=\mathrm{N}$. The compound showed two triplets at appropriate signals and chemical shifts in ${ }^{1} \mathrm{H}-\mathrm{NMR}$ spectra at around $\delta 3.0(J=7.5 \mathrm{~Hz})$ and $2.5(J=7.8 \mathrm{~Hz})$ and ${ }^{13} \mathrm{C}$-NMR spectra showed at around $\delta 30.1$ and 22.2 which could be accounted for two methylene groups $\left(-\mathrm{CH}_{2}-\mathrm{CH}_{2}-\right)$ forming a linker chain through which benzimidazole ring attached with hydrazide. The presence of two doublet and one triplet at around $\delta 7.6(J=7.8 \mathrm{~Hz}), 7.5(J=7.2 \mathrm{~Hz})$ and $7.2(J=7.5 \mathrm{~Hz})$ indicative of benzimidazole hydrogen. The mass spectrum exhibited molecular ion peak at $m / z 232$ according to their molecular formula $\mathrm{C}_{11} \mathrm{H}_{12} \mathrm{~N}_{4} \mathrm{O}_{2}$. In third step, different types of acids were reacted with compound 3to get 1-(1H-benzo[d]imidazol-2yl)-3-(5-(chloromethyl)-1,3,4-oxadiazol-2-yl) propan-1-one (4). In fourth step the compound $\mathbf{4}$ was further treated with different types of secondary amines in presence of sodium acetate to furnish new 1-(1H-benzo[d]imidazol-2-yl)-3-(5(methyl substituted)-1,3,4-oxadiazol-2-yl)propan-1-one (4a-1).

In general, IR spectra of the compounds showed peaks at $3326 \mathrm{~cm}^{-1}$ for $\mathrm{N}-H$. In ${ }^{1} \mathrm{H}$-NMR spectra, there appeared a singlet at around $\delta 12.3$ indicative of ring $\mathrm{N}-H$ and it disappeared by addition of $\mathrm{D}_{2} \mathrm{O}$ as confirmation for this group. The appeared peak in IR spectra at around 1724 and $1660 \mathrm{~cm}^{-1}$ accounted for $\mathrm{C}=\mathrm{O}$ and $\mathrm{C}=\mathrm{N}$ group. The chemical shift in ${ }^{13} \mathrm{C}-\mathrm{NMR}$ spectra at $\delta 173.1$ and 154.9 could be accounted for $\mathrm{C}=\mathrm{O}$ and $\mathrm{C}=\mathrm{N}$ group. The characteristic peaks at around 1384 and $1028 \mathrm{~cm}^{-1}$ for $\mathrm{N}-\mathrm{N}=\mathrm{C}$ and $\mathrm{C}-\mathrm{O}-\mathrm{C}$ as indicative the formation of oxadiazole ring. All the synthetic compounds showed two triplets at appropriate signals and chemical shifts in ${ }^{1} \mathrm{H}-\mathrm{NMR}$ spectra at around $\delta 2.8(\mathrm{~J}=6.9 \mathrm{~Hz}), 3.2$ $(J=7.2 \mathrm{~Hz})$ and ${ }^{13} \mathrm{C}-\mathrm{NMR}$ spectra showed at around $\delta 30.2$ and 27.9 which could be accounted for two methylene group $\left(-\mathrm{CH}_{2}-\mathrm{CH}_{2}-\right)$ forming a linker chain through which benzimidazole attached with oxadiazole ring made up the complete back bone of all the synthetic compounds. The presence of two doublet and one triplet at around $\delta 7.6(J=7.8 \mathrm{~Hz}), 7.4(J=7.5 \mathrm{~Hz})$ and $7.2(J=7.5 \mathrm{~Hz})$ indicative of benzimidazole hydrogen. The signals in ${ }^{13} \mathrm{C}$-NMR spectra which appeared at around $\delta 161.1$ and 159.1 could be for oxadiazole carbon ring. Other peaks were observed at appropriate $\delta$ values supporting the structure of compounds. The mass spectra (ESI-MS) showed the presence of peak at definite $\mathrm{m} / \mathrm{z}$ value in accordance to the molecular ion peak.

\subsection{In-silico data prediction}

\subsubsection{Physicochemical properties prediction}

Molecular descriptors and drug likeliness properties of compounds were analyzed by using Molinspiration server based on Lipinski rules of five. Lipinski's rule of five is commonly used by pharmaceutical chemists in drug design and development to predict oral bioavailability of potential lead or drug molecules. The rule states that most drug-like molecules have $\log \mathrm{P}<=5$, molecular weight $<=500$ daltons, number of hydrogen bond acceptors $<=10$ and number of hydrogen bond donors $<=5$. Molecules violating more than one of these rules may have problems with bioavailability. Physicochemical parameters play a vital role in generation and escalation of bioactivity of chemical entity [33-34].

Molinspirationa web based software was used to acquire parameter such as Log P, TPSA, drug likeness. LogP (octanol/water partition coefficient), which are necessary in QSAR studies and rational drug design as a measure of molecular hydrophobicity. Hydrophobicity affects drug absorption, bioavailability, hydrophobic drug-receptor interactions, metabolism of molecules, as well as their toxicity. It is calculated by the methodology developed by Molinspiration as a sum of fragment based contributions and correction factors used to predict the permeability of molecule across the cell membrane. Topological polar surface area (TPSA) is a very useful parameter for prediction of drug transport properties. Polar surface area is defined as a sum of surfaces of polar atoms (usually oxygen, nitrogen and attached hydrogen) in a molecule. The absorption percentage $(\% \mathrm{Ab})$ was also calculated by using the following formula: \% Ab = $109-[0: 345 \times$ TPSA] (Table 3) [36-40].

The molecular descriptors of synthetic compounds were tested with Lipinski's rule of five, interestingly all the ligands have molecular weight in range of 330 450 ( $<500)$. Low molecular weight drug molecules $(<500)$ are easily transported. Diffuse and absorbed as compared to heavy molecules. Molecular weight is an important aspect in therapeutic drug action, if it increases correspondingly it effects the drug action. Number of hydrogen bond donors $(\mathrm{NH}$ and $\mathrm{OH}$ ) in the tested compounds were found to be within Lipinski limit range that is less than 5 and number of hydrogen bond acceptors ( $\mathrm{O}$ and $\mathrm{N}$ atoms) were also found within the limit that is less than 10 except compound $\mathbf{4 e}, \mathbf{4 f}, \mathbf{4 g}, \mathbf{4 i}, \mathbf{4 k}$ and 4l. Lipophilicity ( $\log \mathrm{P}$ value) and TPSA values are two important properties for the prediction of oral bioavailability for drug molecules. Topological polar surface area (TPSA) was calculated and found within the range. $\mathrm{O}^{-}$and $\mathrm{N}^{-}$ centered as polar fragment were considered. TPSA has shown to be a very good descriptor and characterizing drug absorption including intestinal absorption, bioavailability, Caco-2 permeability and BBB penetration. The highest degree of lipophilicity was found with the compounds, which are an indication for good lipid solubility that will help the drug to interact with membranes. TPSA was calculated from the surface areas that are occupied by oxygen and nitrogen atoms and hydrogen atoms attached to them. Thus, the TPSA is closely related to hydrogen bonding potential of a compound. In the present study, all compound were exhibited $87.92-139.45 \AA$ value of TPSA, indicates good bioavailability by oral route except $\mathbf{4 e}, \mathbf{4 f}, \mathbf{4 g}$ and $\mathbf{4 l}$. Good bioavailability is more likely for compounds with $\leq 10$ rotatable bonds and TPSA of $\leq 140 \AA$. As the number of rotatable bonds increases, the molecule becomes more flexible and more adaptable for efficient interaction with a particular binding pocket. Interestingly all compounds have 7-8 rotatable bonds and flexible within the Lipinski limit [41-44].

The calculated molecular properties of compounds $(\mathbf{4 a - 1})$ were presented in Table 3 and 4 . The compounds did not violate Lipinski's rule of five and Ghose filter, however zero violation was observed for compounds except $4 \mathbf{e}, \mathbf{4 f}, \mathbf{4 g}, \mathbf{4 i}$, $4 \mathbf{k}$ and $\mathbf{4 l}$ compound (one violation detected). The compounds were showed 0-1 violations and are expected to be orally active. Molecular hydrophobicity or lipophilicity is indicated by $\log \mathrm{P}$ or partition coefficient. $\log \mathrm{P}$ values of the compounds were found to be less than 5 and are in not violation of Lipinski's rule of five and Ghose filter, suggesting good permeability across cell membrane. Molecular weight of compounds were found to be less than 500 and thus these molecules are anticipated to be easily transported, diffused and absorbed in compare to large molecules. Numbers of hydrogen bond donors $(\mathrm{NH}$ and $\mathrm{OH})$ in 
the synthetic compounds were found in accordance with Lipinski's rule of five that is less than 5. TPSA of compound was observed in the range of 87.92 $139.45 \AA$ and that is below the limit of $160 \AA$. The percentages of absorption for the compounds calculated from TPSA were found in the range 51.38-78.67 and indicated good oral bioavailability (Table 3). The molar refractivity of compounds was found in the range 93.94-107.09 except compound $\mathbf{4 b}$ (134.53) that is obeyed Ghose filter (Table 4).

Table 3: Lipinski's rule of five predicted physicochemical properties of compounds (4a-l).

\begin{tabular}{|c|c|c|c|c|c|c|c|c|c|c|}
\hline Compd. & $\%$ Abs & $\operatorname{miLog} P_{o} / w$ & TPSA $\left(\mathbf{A}^{0}\right)$ & $\mathrm{n}$ atoms & MW & n-ON & n-OHNH & n-violation & n-rotb & MV \\
\hline RO5 & & $<5$ & & & $<500$ & $<10$ & $<5$ & $\leq 1$ & & \\
\hline $4 a$ & 78.67 & 0.74 & 87.92 & 24 & 327.39 & 7 & 1 & 0 & 8 & 302.43 \\
\hline $4 b$ & 78.67 & 3.39 & 87.92 & 32 & 423.48 & 7 & 1 & 0 & 8 & 378.52 \\
\hline $4 c$ & 73.40 & -0.36 & 103.18 & 27 & 369.43 & 9 & 2 & 0 & 7 & 333.81 \\
\hline $4 d$ & 62.15 & 0.31 & 135.78 & 31 & 416.40 & 10 & 2 & 0 & 7 & 346.64 \\
\hline $4 e$ & 56.70 & -1.42 & 151.57 & 28 & 381.35 & 11 & 3 & 1 & 7 & 315.05 \\
\hline $4 f$ & 51.38 & -0.29 & 166.34 & 30 & 404.39 & 12 & 4 & 1 & 7 & 334.91 \\
\hline $4 g$ & 53.70 & -1.25 & 160.28 & 30 & 405.38 & 12 & 3 & 1 & 7 & 331.74 \\
\hline $4 h$ & 65.04 & -1.13 & 127.42 & 25 & 338.33 & 10 & 2 & 0 & 7 & 283.78 \\
\hline $4 i$ & 60.88 & -1.37 & 139.45 & 26 & 353.35 & 11 & 3 & 1 & 8 & 296.19 \\
\hline $4 j$ & 69.48 & -0.60 & 114.53 & 25 & 337.34 & 9 & 2 & 0 & 7 & 287.94 \\
\hline $4 k$ & 58.59 & -0.01 & 146.11 & 29 & 397.39 & 11 & 3 & 1 & 7 & 337.82 \\
\hline 41 & 54.62 & -1.04 & 157.62 & 28 & 380.37 & 11 & 4 & 1 & 7 & 318.21 \\
\hline
\end{tabular}

\%Abs: Percentage of absorption, TPSA: Topological polar surface area, $\mathrm{n}$ atoms: Number of atoms, $\mathrm{n}$-rotb: Number of rotatable bonds, MW: Molecular weight, MV: Molecular volume, miLogP: Logarithm of partition coefficient between n-octanol and water, $n$-OHNH: Number of hydrogen bond donors, $n$-ON: Number of hydrogen bond acceptors, n violations: Number of "Rule-of-five" violation, RO5: Rule of five.

Table 4: Ghose filter predicted drug likeness properties of compounds (4a-1).

\begin{tabular}{|c|c|c|c|c|c|c|}
\hline Compd. & $\log P$ & Molar Refractivity & Molecular Weight & Number of atoms & Polar Surface Area & Color Indication \\
\hline Ghose filter & $-0.4-5.6$ & $40-130$ & $160-480$ & $20-70$ & $<140$ & Green \\
\hline $4 a$ & 1.883 & 93.94 & 306 & 24 & 66.62 & Green \\
\hline $4 b$ & 3.431 & 134.53 & 402 & 32 & 66.62 & Pink \\
\hline $4 c$ & 0.606 & 104.71 & 346 & 27 & 69.86 & Green \\
\hline 4d & 1.506 & 116.21 & 400 & 31 & 100.76 & Green \\
\hline $4 e$ & 1.227 & 98.73 & 366 & 28 & 100.76 & Green \\
\hline $4 f$ & -0.576 & 108.15 & 388 & 30 & 103.7 & Green \\
\hline $4 g$ & 0.806 & 107.09 & 390 & 30 & 108.41 & Green \\
\hline $4 h$ & 1.038 & 91.77 & 324 & 25 & 91.34 & Green \\
\hline $4 \mathbf{i}$ & 1.362 & 95.07 & 338 & 26 & 91.34 & Green \\
\hline $4 \mathbf{j}$ & 0.673 & 93.25 & 322 & 25 & 78.98 & Green \\
\hline $4 k$ & 1.235 & 103.31 & 378 & 29 & 100.76 & Green \\
\hline 41 & 0.722 & 99.79 & 364 & 28 & 96.05 & Green \\
\hline
\end{tabular}

\subsubsection{The score of bioactivity}

The compounds were also checked for bioactivity by calculating activity score for different target such as GPCR (G-protein coupled receptor) ligand, ion channel modulator, kinase inhibitor, nuclear receptor ligand [29, 45-46]. The compounds were showed good GPCR ligand affinity and compound $\mathbf{4 c}, \mathbf{4 f}$ and 4k showed excellent GPCR ligand affinity. Compounds were also showed good enzyme inhibitor and compound $\mathbf{4 d}, \mathbf{4 f}, \mathbf{4 h}, \mathbf{4} \mathbf{j}$ and $\mathbf{4 l}$ were found excellent enzyme inhibitor activity. Compounds $\mathbf{4 k}$ and $\mathbf{4 l}$ showed good protease inhibition and compounds $\mathbf{4 f}$ showed better kinase inhibitor. The obtained result indicate that compound $\mathbf{4 f}$ has better Enzyme inhibitor > GPCR ligand $>$ Kinase inhibitor >Protease inhibitor >Ion channel modulator> Nuclear receptor ligand and compound $4 \mathbf{k}$ are found better ligand for GPCR ligand >Protease inhibitor>enzyme inhibitor> Ion channel modulator $>$ Kinase inhibitor $>$ Nuclear receptor ligand. Compound $\mathbf{4} \mathbf{v}$ is also shown better ligand for enzyme inhibition and GPCR ligand as per in-silico data prediction (Enzyme inhibitor > GPCR ligand $>$ Kinase inhibitor $>$ Protease inhibitor $>$ Ion channel modulator $>$ Nuclear receptor ligand). A molecule having bioactivity score more than 0.00 is most likely to exhibit considerable biological activities, while values -0.50 to 0.00 are expected to be moderately active and if score is less than -0.50 it is presumed to be inactive [45-48]. The most promising compounds as per the bioactivity scores were identified such as $3 \mathbf{i i i}$, $4 \mathbf{i i i}$ and $\mathbf{4 v}$ which are predicted to act by more than three mechanisms (Table 5). 
J. Chil. Chem. Soc., 66, №2 (2021)

Table 5: Predicted bioactivity score of compounds (4a-I).

\begin{tabular}{|c|c|c|c|c|c|c|c|}
\hline Compd. & 3D Structure & $\begin{array}{l}\text { GPCR } \\
\text { ligand }\end{array}$ & $\begin{array}{l}\text { Ion channel } \\
\text { modulator }\end{array}$ & $\begin{array}{c}\text { Kinase } \\
\text { inhibitor }\end{array}$ & $\begin{array}{c}\text { Nuclear } \\
\text { receptor ligand }\end{array}$ & $\begin{array}{l}\text { Protease } \\
\text { inhibitor }\end{array}$ & $\begin{array}{l}\text { Enzyme } \\
\text { inhibitor }\end{array}$ \\
\hline $4 a$ & & 0.15 & -0.12 & -0.16 & -0.51 & 0.04 & 0.12 \\
\hline $4 b$ & & 0.13 & -0.05 & -0.05 & -0.14 & 0.03 & 0.18 \\
\hline $4 c$ & & 0.25 & -0.10 & -0.02 & -0.55 & 0.08 & 0.10 \\
\hline $4 d$ & & 0.06 & -0.29 & -0.12 & -0.56 & -0.02 & 0.22 \\
\hline $4 e$ & & -0.01 & -0.42 & -0.34 & -0.73 & -0.06 & 0.1 \\
\hline $4 f$ & & 0.29 & -0.12 & 0.26 & -0.95 & -0.00 & 0.48 \\
\hline $4 g$ & & 0.08 & -0.48 & -0.20 & -1.10 & -0.30 & 0.14 \\
\hline $4 h$ & & 0.08 & -0.28 & -0.13 & -0.65 & 0.01 & 0.24 \\
\hline $4 i$ & & 0.07 & -0.19 & -0.16 & -0.56 & 0.02 & 0.22 \\
\hline $4 j$ & & 0.17 & -0.20 & 0.01 & -0.61 & -0.00 & 0.45 \\
\hline $4 k$ & & 0.27 & -0.10 & -0.27 & -0.55 & 0.21 & 0.14 \\
\hline 41 & & 0.12 & -0.38 & -0.00 & -0.88 & 0.11 & 0.30 \\
\hline
\end{tabular}

GPCR= G-protein coupled receptor, $>0$ - active, $-5.0-0.0$ - moderately active, $<-5.0$ - inactive. 


\subsubsection{Osiris property explorer}

Osiris property explorer was used to determine pharmacokinetic parameters such as toxicity potential, solubility, overall drug-likeness and drug score of compounds (www.organicchemistry. org/prog/peo/). The results of virtual screening are valued and color coded either green/red/ yellow for properties such as effect on mutagenicity, reproductive system, irritant effect and tumorigenicity. Properties shown in red color indicate high risks of undesired effects while a green color showed drug conform behavior, compatibility and safety in-vivo
[52-53]. This program predicts on the basis of functional group similarity of investigated compound with the extensively in-vitro and in-vivo studied compounds present in its database. The results clearly indicated that all compounds showed green color except 4a (yellow color for Irritant effect) would be safe and expected to show no toxicity regarding tumorigenicity, mutagenicity, irritant effect and effect on reproductive system. Results of toxicity risks, drug likeness score and drug score of compounds were presented in Table 6.

Table 6: Osiris property explorer toxicity and drug-relevant properties of compounds (4a-1).

\begin{tabular}{|c|c|c|c|c|c|c|c|c|}
\hline \multirow[t]{2}{*}{ Compd. } & \multicolumn{4}{|c|}{ Toxicity } & \multicolumn{4}{|c|}{ Drug-relevant properties } \\
\hline & Tumorigenic & $\begin{array}{c}\text { Reproductive } \\
\text { effect }\end{array}$ & $\begin{array}{c}\text { Irritant } \\
\text { effect }\end{array}$ & Mutagenicity & cLogP & Solubility & $\begin{array}{l}\text { Drug- } \\
\text { likeness }\end{array}$ & $\begin{array}{l}\text { Drug } \\
\text { Score }\end{array}$ \\
\hline $4 \mathbf{a}$ & Green & Green & Yellowish red & Green & 1.31 & -1.47 & 5.41 & 0.55 \\
\hline $4 b$ & Green & Green & Green & Green & 2.85 & -4.73 & 1.18 & 0.56 \\
\hline $4 c$ & Green & Green & Green & Green & -0.85 & -0.53 & 6.87 & 0.91 \\
\hline $4 d$ & Green & Green & Green & Green & 0.24 & -2.69 & 0.54 & 0.67 \\
\hline $4 e$ & Green & Green & Green & Green & -1.58 & -2.04 & 2.85 & 0.86 \\
\hline $4 f$ & Green & Green & Green & Green & 0.03 & -5.47 & -0.7 & 0.4 \\
\hline $4 \mathrm{~g}$ & Green & Green & Green & Green & -1.41 & -2.36 & 5.13 & 0.85 \\
\hline $4 h$ & Green & Green & Green & Green & 0.26 & -2.7 & 3.68 & 0.88 \\
\hline $4 i$ & Green & Green & Green & Green & -0.15 & -2.89 & 3.68 & 0.86 \\
\hline $4 \mathbf{j}$ & Green & Green & Green & Green & 0.2 & -3.63 & 3.85 & 0.83 \\
\hline $4 k$ & Green & Green & Green & Green & -1.04 & -1.98 & 5.97 & 0.86 \\
\hline 41 & Green & Green & Green & Green & -2.07 & -2.18 & -0.87 & 0.57 \\
\hline
\end{tabular}

Green color indicates no risk or low risk, yellow color medium risk and red color high toxicity risk.

\subsubsection{ADME and Toxicity prediction}

PreADMET server was used for prediction of ADME (Absorption, Distribution, Metabolism and Excretion) and toxicity profile of compounds. Oral dosing is quiet most prefer route for drug administration and therefore to get optimum therapeutic efficacy, intestinal absorption of drug is the first criteria to be considered. Lipinski's rule of five identified some critical property continued indeed the general requirement for absorption of drug molecule. LogP, TPSA, $\mathrm{MW}, \mathrm{nON}, \mathrm{nOHNH}$, nviolation, nrotb and MV data revealed that compounds retain drugs like property and fulfilling the rule of five and to exhibit optimum absorption and bioavailability [61-63]. For accurate and effective prediction of intestinal absorption, several in-vitro methods have been developed. Among them, the most popular cell based model for intestinal permeability is Caco-2 cell system [39]. P-glycoprotein (P-gp) activity in the apical cell membrane may limit the bioavailability of drug molecule [74].

\subsubsection{ADME/T Properties Prediction}

HIA (Human intestinal absorption), BBB (Blood-brain barrier) penetration, Caco-2 cell permeability and Ames test were calculated by Pre ADMET server $[37,39,63]$. The result from Pre ADMET server revealed that the compound 4a, 4b and 4d shown better human intestinal absorption (HIA) score. More HIA values indicate that the compound could be better absorbed from the intestinal tract upon oral administration. AMES toxicity test was used to identify that a compound is mutagenic or not. The tested compound displayed negative AMES toxicity test, which means that maximum ligands showed negative [75-76]. It is apparent that from the toxicity prediction study that the designed compound predicted negative test for carcinogenicity (Mouse) and to be carcinogenic (in Rat) and outcomes results are presented in Table 7. 
J. Chil. Chem. Soc., 66, N² (2021)

Table 7: Toxicity prediction by Pre ADMET tool of compounds (4a-1).

\begin{tabular}{|c|c|c|c|}
\hline Compd. & Toxicity prediction & Name of Test & Values of Test \\
\hline \multirow[t]{6}{*}{$4 \mathbf{4 a}$} & \multirow[t]{4}{*}{ Ames test } & Ames TA100 (+S9) & - \\
\hline & & Ames TA100 (-S9) & - \\
\hline & & Ames TA1535 (+S9) & - \\
\hline & & Ames TA1535 (-S9) & + \\
\hline & \multirow[t]{2}{*}{ Carcinogenicity } & Carcinogenicity (Mouse) & - \\
\hline & & Carcinogenicity (Rat) & + \\
\hline \multirow[t]{6}{*}{$4 b$} & \multirow[t]{4}{*}{ Ames test } & Ames TA100 (+S9) & - \\
\hline & & Ames TA100 (-S9) & - \\
\hline & & Ames TA1535 (+S9) & - \\
\hline & & Ames TA1535 (-S9) & - \\
\hline & \multirow[t]{2}{*}{ Carcinogenicity } & Carcinogenicity (Mouse) & - \\
\hline & & Carcinogenicity (Rat) & + \\
\hline \multirow[t]{6}{*}{$4 c$} & \multirow[t]{4}{*}{ Ames test } & Ames TA100 (+S9) & - \\
\hline & & Ames TA100 (-S9) & - \\
\hline & & Ames TA1535 (+S9) & - \\
\hline & & Ames TA1535 (-S9) & - \\
\hline & \multirow[t]{2}{*}{ Carcinogenicity } & Carcinogenicity (Mouse) & - \\
\hline & & Carcinogenicity (Rat) & + \\
\hline \multirow[t]{6}{*}{ 4d } & \multirow[t]{4}{*}{ Ames test } & Ames TA100 (+S9) & + \\
\hline & & Ames TA100 (-S9) & + \\
\hline & & Ames TA1535 (+S9) & - \\
\hline & & Ames TA1535 (-S9) & - \\
\hline & \multirow[t]{2}{*}{ Carcinogenicity } & Carcinogenicity (Mouse) & - \\
\hline & & Carcinogenicity (Rat) & + \\
\hline \multirow[t]{6}{*}{$4 e$} & \multirow[t]{4}{*}{ Ames test } & Ames TA100 (+S9) & - \\
\hline & & Ames TA100 (-S9) & + \\
\hline & & Ames TA1535 (+S9) & - \\
\hline & & Ames TA1535 (-S9) & + \\
\hline & Carcinogenicity & Carcinogenicity (Mouse) & - \\
\hline & & Carcinogenicity (Rat) & + \\
\hline $4 f$ & Ames test & Ames TA100 (+S9) & - \\
\hline & & Ames TA100 (-S9) & + \\
\hline & & Ames TA1535 (+S9) & - \\
\hline & & Ames TA1535 (-S9) & - \\
\hline & Carcinogenicity & Carcinogenicity (Mouse) & - \\
\hline & & Carcinogenicity (Rat) & + \\
\hline $4 \mathrm{~g}$ & Ames test & Ames TA100 (+S9) & - \\
\hline & & Ames TA100 (-S9) & + \\
\hline & & Ames TA1535 (+S9) & - \\
\hline & & Ames TA1535 (-S9) & + \\
\hline & Carcinogenicity & Carcinogenicity (Mouse) & - \\
\hline & & Carcinogenicity (Rat) & + \\
\hline $4 h$ & Ames test & Ames TA100 (+S9) & + \\
\hline & & Ames TA100 (-S9) & + \\
\hline & & Ames TA1535 (+S9) & - \\
\hline & & Ames TA1535 (-S9) & + \\
\hline & Carcinogenicity & Carcinogenicity (Mouse) & - \\
\hline & & Carcinogenicity (Rat) & + \\
\hline $4 i$ & Ames test & Ames TA100 (+S9) & + \\
\hline & & Ames TA100 (-S9) & + \\
\hline & & Ames TA1535 (+S9) & - \\
\hline & & Ames TA1535 (-S9) & + \\
\hline & Carcinogenicity & Carcinogenicity (Mouse) & - \\
\hline & & Carcinogenicity (Rat) & + \\
\hline $4 j$ & Ames test & Ames TA100 (+S9) & - \\
\hline & & Ames TA100 (-S9) & + \\
\hline & & Ames TA1535 (+S9) & - \\
\hline & & Ames TA1535 (-S9) & + \\
\hline & Carcinogenicity & Carcinogenicity (Mouse) & - \\
\hline & & Carcinogenicity (Rat) & + \\
\hline $4 k$ & Ames test & Ames TA100 (+S9) & - \\
\hline & & Ames TA100 (-S9) & - \\
\hline & & Ames TA1535 (+S9) & - \\
\hline & & Ames TA1535 (-S9) & - \\
\hline & Carcinogenicity & Carcinogenicity (Mouse) & - \\
\hline & & Carcinogenicity (Rat) & + \\
\hline
\end{tabular}




\begin{tabular}{|c|c|c|c|}
\hline \multirow{4}{*}{$4 \mathbf{* 1}$ Ames test } & Ames TA100 (+S9) & + \\
\cline { 3 - 4 } & & Ames TA100 (-S9) & + \\
\cline { 3 - 4 } & & Ames TA1535 (+S9) & - \\
\cline { 3 - 4 } & & Ames TA1535 (-S9) & + \\
\cline { 2 - 4 } & Carcinogenicity & Carcinogenicity (Mouse) & - \\
& & Carcinogenicity (Rat) & + \\
\hline
\end{tabular}

-: Negative; +: Positive

These constraints have been shown to associate very well with the human intestinal absorption, Caco-2 mono layers permeability and blood-brain barrier penetration. The compound which has less polar chemical structure was predicted to cross BBB and getting data is presented in Table 8. Molecular polar surface area (MPSA) is calculated based on the methodology published by Ertl [34] as a sum of fragment based contributions in which $\mathrm{O}^{-}$and $\mathrm{N}^{-}$centered polar fragments are to be measured and calculated by surface areas that employed by oxygen, nitrogen and active hydrogen atoms attached to them. Molecular volume (MV) governs the transport characteristics of molecules, such as intestinal absorption or blood brain barrier penetration. Molecular volume is therefore often used in QSAR studies to model molecular properties and biological activity. Method for the calculation of molecular volume developed at Molinspiration is based on group contributions. Number of rotatable bonds (nrotb) is a simple topological parameter that measures molecular flexibility..

Table 8: ADME/T prediction by Pre ADMET server of compounds (4a-l).

\begin{tabular}{|c|c|c|c|}
\hline Compd. & Toxicity prediction & Name of Test & Values of Test \\
\hline \multirow[t]{8}{*}{$\mathbf{4 a}$} & \multirow[t]{4}{*}{ Absorption } & HIA, \% (Human intestinal absorption) & 92.215603 \\
\hline & & Caco-2 cell permeability in $\mathrm{nm} / \mathrm{sec}$ ( In-vitro) & 15.7825 \\
\hline & & MDCK cell permeability in $\mathrm{nm} / \mathrm{sec}$ ( In-vitro) & 215.717 \\
\hline & & Skin permeability $(\log K \mathrm{p})$ in $\mathrm{cm} / \mathrm{hour}$ ( In-vitro) & -4.11061 \\
\hline & \multirow[t]{2}{*}{ Bioavailability } & Buffer solubility in $\mathrm{mg} / \mathrm{ml}$ & 367.222 \\
\hline & & Pure water solubility in $\mathrm{mg} / \mathrm{ml}$ & 1247.44 \\
\hline & \multirow[t]{2}{*}{ Distribution } & Plasma protein binding \% ( In-vitro) & 47.279628 \\
\hline & & $\begin{array}{l}\text { Blood-brain barrier penetration ( In-vivo) } \\
\text { (C. brain/C. blood) }\end{array}$ & 0.0493072 \\
\hline \multirow[t]{8}{*}{ 4b } & \multirow[t]{4}{*}{ Absorption } & HIA, \% (Human intestinal absorption) & 95.342099 \\
\hline & & Caco-2 cell permeability in $\mathrm{nm} / \mathrm{sec}$ ( In-vitro) & 27.4034 \\
\hline & & MDCK cell permeability in $\mathrm{nm} / \mathrm{sec}$ ( In-vitro) & 37.0983 \\
\hline & & Skin permeability $(\operatorname{logKp})$ in $\mathrm{cm} /$ hour $($ In-vitro $)$ & -2.86615 \\
\hline & \multirow[t]{2}{*}{ Bioavailability } & Buffer solubility in $\mathrm{mg} / \mathrm{ml}$ & 4.12198 \\
\hline & & Pure water solubility in $\mathrm{mg} / \mathrm{ml}$ & 0.389741 \\
\hline & \multirow[t]{2}{*}{ Distribution } & Plasma protein binding \% ( In-vitro) & 95.889629 \\
\hline & & $\begin{array}{l}\text { Blood-brain barrier penetration ( In-vivo) } \\
\text { (C. brain/C. blood) }\end{array}$ & 0.207344 \\
\hline \multirow[t]{8}{*}{$4 c$} & \multirow[t]{4}{*}{ Absorption } & HIA, \% (Human intestinal absorption) & 88.872345 \\
\hline & & Caco-2 cell permeability in $\mathrm{nm} / \mathrm{sec}$ ( In-vitro) & 7.42263 \\
\hline & & MDCK cell permeability in $\mathrm{nm} / \mathrm{sec}$ ( In-vitro) & 2.63357 \\
\hline & & Skin permeability $(\operatorname{logKp})$ in $\mathrm{cm} /$ hour $($ In-vitro $)$ & -4.98237 \\
\hline & \multirow[t]{2}{*}{ Bioavailability } & Buffer solubility in $\mathrm{mg} / \mathrm{ml}$ & 1267.96 \\
\hline & & Pure water solubility in $\mathrm{mg} / \mathrm{ml}$ & 1661.14 \\
\hline & \multirow[t]{2}{*}{ Distribution } & Plasma protein binding \% ( In-vitro) & 28.649417 \\
\hline & & $\begin{array}{l}\text { Blood-brain barrier penetration ( In-vivo) } \\
\text { (C. brain/C. blood) }\end{array}$ & 0.0584475 \\
\hline \multirow[t]{8}{*}{ 4d } & \multirow[t]{4}{*}{ Absorption } & HIA, \% (Human intestinal absorption) & 91.308684 \\
\hline & & Caco-2 cell permeability in $\mathrm{nm} / \mathrm{sec}$ ( In-vitro) & 1.13618 \\
\hline & & MDCK cell permeability in $\mathrm{nm} / \mathrm{sec}$ ( In-vitro) & 2.52908 \\
\hline & & Skin permeability $(\log K \mathrm{p})$ in $\mathrm{cm} / \mathrm{hour}($ In-vitro $)$ & -4.95527 \\
\hline & \multirow[t]{2}{*}{ Bioavailability } & Buffer solubility in $\mathrm{mg} / \mathrm{ml}$ & 3.02145 \\
\hline & & Pure water solubility in $\mathrm{mg} / \mathrm{ml}$ & 0.346366 \\
\hline & \multirow[t]{2}{*}{ Distribution } & Plasma protein binding $\%$ ( In-vitro $)$ & 80.038452 \\
\hline & & $\begin{array}{l}\text { Blood-brain barrier penetration ( In-vivo) } \\
\text { (C. brain/C. blood) }\end{array}$ & 0.0465817 \\
\hline \multirow[t]{8}{*}{$4 e$} & \multirow[t]{4}{*}{ Absorption } & HIA, $\%$ (Human intestinal absorption) & 75.207209 \\
\hline & & Caco-2 cell permeability in $\mathrm{nm} / \mathrm{sec}$ ( In-vitro) & 1.70306 \\
\hline & & MDCK cell permeability in $\mathrm{nm} / \mathrm{sec}$ ( In-vitro) & 0.833755 \\
\hline & & Skin permeability $(\operatorname{logKp})$ in $\mathrm{cm} /$ hour $($ In-vitro $)$ & -5.12146 \\
\hline & \multirow[t]{2}{*}{ Bioavailability } & Buffer solubility in $\mathrm{mg} / \mathrm{ml}$ & 185.214 \\
\hline & & Pure water solubility in $\mathrm{mg} / \mathrm{ml}$ & 154.972 \\
\hline & \multirow[t]{2}{*}{ Distribution } & Plasma protein binding $\%$ ( In-vitro) & 54.013105 \\
\hline & & $\begin{array}{l}\text { Blood-brain barrier penetration ( In-vivo) } \\
\text { (C. brain/C. blood) }\end{array}$ & 0.0420603 \\
\hline \multirow[t]{3}{*}{ 4f } & \multirow[t]{3}{*}{ Absorption } & HIA, \% (Human intestinal absorption) & 83.700104 \\
\hline & & Caco-2 cell permeability in $\mathrm{nm} / \mathrm{sec}$ ( In-vitro) & 1.7489 \\
\hline & & MDCK cell permeability in $\mathrm{nm} / \mathrm{sec}$ ( In-vitro) & 0.687794 \\
\hline
\end{tabular}




\begin{tabular}{|c|c|c|c|}
\hline & & Skin permeability $(\log \mathrm{Kp})$ in $\mathrm{cm} /$ hour ( In-vitro) & -5.1046 \\
\hline & \multirow[t]{2}{*}{ Bioavailability } & Buffer solubility in $\mathrm{mg} / \mathrm{ml}$ & 28.2703 \\
\hline & & Pure water solubility in $\mathrm{mg} / \mathrm{ml}$ & 4.12212 \\
\hline & \multirow[t]{2}{*}{ Distribution } & Plasma protein binding \% ( In-vitro) & 60.886301 \\
\hline & & $\begin{array}{l}\text { Blood-brain barrier penetration ( In-vivo) } \\
\text { (C. brain/C. blood) }\end{array}$ & 0.0549292 \\
\hline \multirow[t]{8}{*}{$4 \mathrm{~g}$} & \multirow[t]{4}{*}{ Absorption } & HIA, \% (Human intestinal absorption) & 73.189010 \\
\hline & & Caco-2 cell permeability in $\mathrm{nm} / \mathrm{sec}$ ( In-vitro) & 0.943094 \\
\hline & & MDCK cell permeability in $\mathrm{nm} / \mathrm{sec}$ ( In-vitro) & 0.457272 \\
\hline & & Skin permeability $(\log \mathrm{Kp})$ in $\mathrm{cm} /$ hour ( In-vitro) & -5.22165 \\
\hline & \multirow[t]{2}{*}{ Bioavailability } & Buffer solubility in $\mathrm{mg} / \mathrm{ml}$ & 10.4568 \\
\hline & & Pure water solubility in $\mathrm{mg} / \mathrm{ml}$ & 41.8636 \\
\hline & \multirow[t]{2}{*}{ Distribution } & Plasma protein binding \% ( In-vitro) & 43.962351 \\
\hline & & $\begin{array}{l}\text { Blood-brain barrier penetration ( In-vivo) } \\
\text { (C. brain/C. blood) }\end{array}$ & 0.0358059 \\
\hline \multirow[t]{8}{*}{ 4h } & \multirow[t]{4}{*}{ Absorption } & HIA, \% (Human intestinal absorption) & 85.778193 \\
\hline & & Caco- 2 cell permeability in $\mathrm{nm} / \mathrm{sec}$ ( In-vitro) & 0.502463 \\
\hline & & MDCK cell permeability in $\mathrm{nm} / \mathrm{sec}$ ( In-vitro) & 2.52258 \\
\hline & & Skin permeability (logKp) in cm/hour ( In-vitro) & -5.0398 \\
\hline & \multirow[t]{2}{*}{ Bioavailability } & Buffer solubility in $\mathrm{mg} / \mathrm{ml}$ & 163.365 \\
\hline & & Pure water solubility in $\mathrm{mg} / \mathrm{ml}$ & 328.207 \\
\hline & \multirow[t]{2}{*}{ Distribution } & Plasma protein binding \% ( In-vitro) & 60.687062 \\
\hline & & $\begin{array}{l}\text { Blood-brain barrier penetration ( In-vivo) } \\
\text { (C. brain/C. blood) }\end{array}$ & 0.0421355 \\
\hline \multirow[t]{8}{*}{$4 \mathbf{i}$} & \multirow[t]{4}{*}{ Absorption } & HIA, \% (Human intestinal absorption) & 77.584349 \\
\hline & & Caco- 2 cell permeability in $\mathrm{nm} / \mathrm{sec}$ ( In-vitro) & 0.51027 \\
\hline & & MDCK cell permeability in $\mathrm{nm} / \mathrm{sec}$ ( In-vitro $)$ & 1.46159 \\
\hline & & Skin permeability $(\operatorname{logKp})$ in $\mathrm{cm} /$ hour ( In-vitro) & -5.06709 \\
\hline & \multirow[t]{2}{*}{ Bioavailability } & Buffer solubility in $\mathrm{mg} / \mathrm{ml}$ & 0.0007629 \\
\hline & & Pure water solubility in $\mathrm{mg} / \mathrm{ml}$ & 1011.94 \\
\hline & \multirow[t]{2}{*}{ Distribution } & Plasma protein binding \% ( In-vitro) & 51.762693 \\
\hline & & $\begin{array}{l}\text { Blood-brain barrier penetration ( In-vivo) } \\
\text { (C. brain/C. blood) }\end{array}$ & 0.0296899 \\
\hline \multirow[t]{8}{*}{$4 \mathbf{j}$} & \multirow[t]{4}{*}{ Absorption } & HIA, \% (Human intestinal absorption) & 88.249693 \\
\hline & & Caco- 2 cell permeability in $\mathrm{nm} / \mathrm{sec}$ ( In-vitro) & 0.797294 \\
\hline & & MDCK cell permeability in $\mathrm{nm} / \mathrm{sec}$ ( In-vitro) & 4.73256 \\
\hline & & Skin permeability (logKp) in cm/hour ( In-vitro) & -4.99595 \\
\hline & \multirow[t]{2}{*}{ Bioavailability } & Buffer solubility in $\mathrm{mg} / \mathrm{ml}$ & 106.264 \\
\hline & & Pure water solubility in $\mathrm{mg} / \mathrm{ml}$ & 431.279 \\
\hline & \multirow[t]{2}{*}{ Distribution } & Plasma protein binding \% ( In-vitro $)$ & 65.290656 \\
\hline & & $\begin{array}{l}\text { Blood-brain barrier penetration ( In-vivo) } \\
\text { (C. brain/C. blood) }\end{array}$ & 0.0466395 \\
\hline \multirow[t]{8}{*}{$4 \mathbf{k}$} & \multirow[t]{4}{*}{ Absorption } & HIA, \% (Human intestinal absorption) & 76.683939 \\
\hline & & Caco-2 cell permeability in $\mathrm{nm} / \mathrm{sec}$ ( In-vitro) & 2.3402 \\
\hline & & MDCK cell permeability in $\mathrm{nm} / \mathrm{sec}($ In-vitro $)$ & 1.91347 \\
\hline & & Skin permeability (logKp) in cm/hour ( In-vitro) & -5.0762 \\
\hline & \multirow[t]{2}{*}{ Bioavailability } & Buffer solubility in $\mathrm{mg} / \mathrm{ml}$ & 346.266 \\
\hline & & Pure water solubility in $\mathrm{mg} / \mathrm{ml}$ & 98.2177 \\
\hline & Distribution & Plasma protein binding \% ( In-vitro) & 57.895070 \\
\hline & & $\begin{array}{l}\text { Blood-brain barrier penetration ( In-vivo) } \\
\text { (C. brain/C. blood) }\end{array}$ & 0.0395919 \\
\hline 41 & Absorption & HIA, \% (Human intestinal absorption) & 75.297321 \\
\hline & & Caco-2 cell permeability in $\mathrm{nm} / \mathrm{sec}$ ( In-vitro) & 2.83561 \\
\hline & & MDCK cell permeability in $\mathrm{nm} / \mathrm{sec}($ In-vitro $)$ & 0.581086 \\
\hline & & Skin permeability (logKp) in cm/hour ( In-vitro) & -5.1312 \\
\hline & Bioavailability & Buffer solubility in $\mathrm{mg} / \mathrm{ml}$ & 435.553 \\
\hline & & Pure water solubility in $\mathrm{mg} / \mathrm{ml}$ & 63.5098 \\
\hline & Distribution & Plasma protein binding \% ( In-vitro) & 38.872860 \\
\hline & & $\begin{array}{l}\text { Blood-brain barrier penetration ( In-vivo) } \\
\text { (C. brain/C. blood) }\end{array}$ & 0.0627287 \\
\hline
\end{tabular}

BBB indicates compounds can cross blood brain barrier, HIA indicates compounds can absorb through intestine; Caco-2 indicates compounds can cross Caco-2 cell. 


\subsubsection{Acute toxicity prediction by T.E.S.T. server (US-EPA)}

T.E.S.T software (version 4.2.1) was used for the prediction of toxicity through molecular features of synthetic compounds as a descriptor for the quantitative structure-activity relationship studies. These molecular features are very important for predicting of QSAR of harmful substances. The acute toxicity such as oral lethal dose for $50 \%$ of test rats (LD50) and mutagenicity of compounds $(\mathbf{4 a}, \mathbf{4 b}, \mathbf{4 c}, \mathbf{4 i}, \mathbf{4 k}$ and $\mathbf{4 l})$ were evaluated by T.E.S.T. software program through a types of method such as Consensus, Hierarchical clustering, FDA and nearest neighbor method. The predicted value of oral rat LD50 was found in the range of $2.30-2.82(-\log 10(\mathrm{~mol} / \mathrm{kg})$ by consensus method, 1.98-2.74 (-Log10 $(\mathrm{mol} / \mathrm{kg})$ by FDA method and 2.25-2.90 $\left(-\log _{10}(\mathrm{~mol} / \mathrm{kg})\right.$ by Nearest neighbor method of compounds. The Mutagenicity value of the compounds was found in the range $0.30-0.67$ by Consensus method, $0.29-0.64$ by Hierarchical clustering Method, $0.28-1.03$ by FDA method and $0.00-0.67$ by Nearest neighbor method and other toxicity results are presented in Table 9.

Table 9: T.E.S.T. software (US-EPA) results of predicted toxicities of compounds (4a-c, $\mathbf{4 i} \& \mathbf{4 k - 1})$.

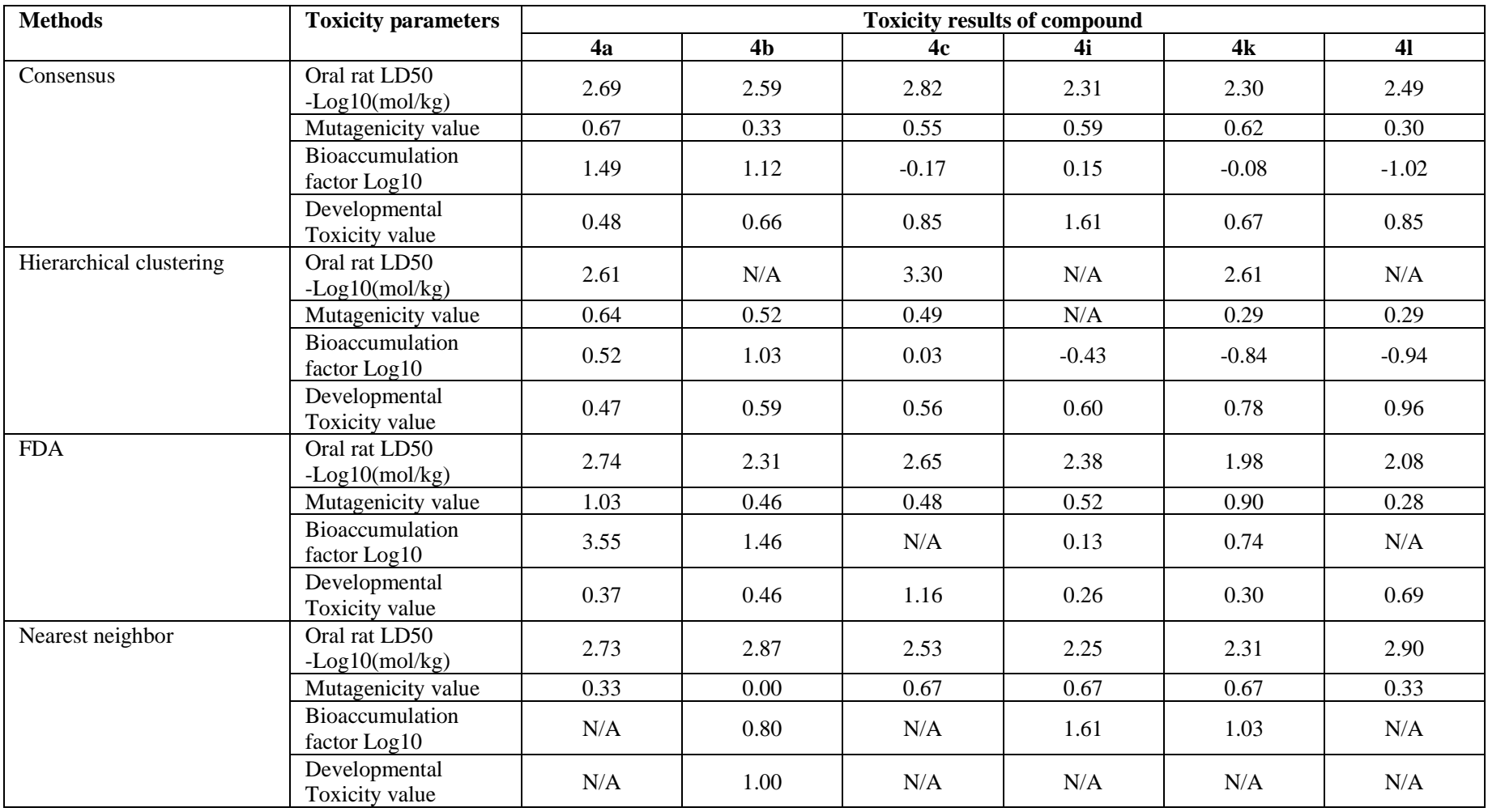

\subsubsection{Metabolism prediction}

The metabolic phase 1 of compound was predicted by Pre ADMET server by setting the strictness of fingerprint matching in default and selecting all models. The most important parameter is cytochrome P450 (CYP 450) which is known as isozymes group and it is involved in metabolism of drugs, fatty acids, steroids, bile acids and carcinogens etc. Some of the cytochrome P450 isoforms could be inhibited by one or more of the tested compounds. Cytochrome P450 enzymes are important enzymes for drug metabolism. Cytochrome P450 enzymes are a family of heme proteins involved in the metabolism of frequent phar- macologically active compounds and can cause drug to drug interactions with co-administered drugs as well as unwanted adverse side effects [66, 77]. In-silico data indicate that the entire designed compounds are substrate for CYP $4503 \mathrm{~A} 4$ except compound $\mathbf{4 l}$ (non substrate). All compounds are found non-substrate for CYP 450 2D6 except compound 4a (substrate) and non inhibitor for CYP 450 2D6 but remarkably compounds $\mathbf{4 h}, \mathbf{4 i}$ and $\mathbf{4 j}$ are inhibitor of CYP $4502 \mathrm{C} 19$, CYP 450 2C9 and CYP $4503 \mathrm{~A} 4$ and substrate for CYP 450 3A4 (Table 10).

Table 10: Cytochrome $\mathrm{P} 450$ enzymes and P-glycoprotein inhibition of compounds (4a-I).

\begin{tabular}{|c|c|c|c|c|c|c|c|}
\hline Compd. & $\begin{array}{c}\text { CYP 450 } \\
\text { 2C19 } \\
\text { Inhibition }\end{array}$ & $\begin{array}{c}\text { CYP 450 } \\
\text { 2C9 } \\
\text { Inhibition }\end{array}$ & $\begin{array}{c}\text { CYP 450 } \\
\text { 2D6 } \\
\text { Inhibition }\end{array}$ & $\begin{array}{c}\text { CYP 450 } \\
\text { 2D6 } \\
\text { Substrate }\end{array}$ & $\begin{array}{c}\text { CYP 450 } \\
\text { 3A4 } \\
\text { Inhibition }\end{array}$ & $\begin{array}{c}\text { CYP 450 } \\
\text { 3A4 } \\
\text { Substrate }\end{array}$ & P-gp inhibitor \\
\hline $4 a$ & Non Inhibitor & Non Inhibitor & Non Inhibitor & Substrate & Non Inhibitor & Substrate & Non Inhibitor \\
\hline $4 c$ & Non Inhibitor & Non Inhibitor & Non Inhibitor & Substrate & Non Inhibitor & Substrate & Non Inhibitor \\
\hline $4 d$ & Inhibitor & Inhibitor & Non Inhibitor & Non Substrate & Inhibitor & Substrate & Non Inhibitor \\
\hline $4 e$ & Non Inhibitor & Inhibitor & Non Inhibitor & Non Substrate & Non Inhibitor & Weakly Substrate & Non Inhibitor \\
\hline $4 \mathrm{~h}$ & Inhibitor & Inhibitor & Non Inhibitor & Non Substrate & Inhibitor & Substrate & Non Inhibitor \\
\hline $4 i$ & Inhibitor & Inhibitor & Non Inhibitor & Non Substrate & Inhibitor & Substrate & Non Inhibitor \\
\hline $4 \mathbf{j}$ & Inhibitor & Inhibitor & Non Inhibitor & Non Substrate & Inhibitor & Substrate & Non Inhibitor \\
\hline $4 k$ & Non Inhibitor & Inhibitor & Non Inhibitor & Non Substrate & Non Inhibitor & Substrate & Non Inhibitor \\
\hline 41 & Non Inhibitor & Inhibitor & Non Inhibitor & Non Substrate & Non Inhibitor & Non Substrate & Non Inhibitor \\
\hline
\end{tabular}

CYP 450; Cytochrome P450 enzyme, P-gp = P-glycoprotein 


\subsubsection{Drug likeness and Violation Prediction for Synthetic Compounds}

Several drug-like rules that defined drug-like characteristics of chemical such as Lipinski's rule, Ghose filter, Lead-like rule, CMC, WDI and MDDR. Pre ADMET server contains drug-likeness prediction module based on these different rules. But the well-known rule for the prediction of drug likeness of newly designed molecules is Lipinski's rule and generally called as rule of five. Another well-known rule is the Lead-like rule that is starting from a quantitative survey based upon 18 lead and drug pairs of chemical structure. Ghose filter outlines drug-likeness limitations as follows: calculated $\log \mathrm{P}$ is between 0.4 and 5.6, molecular weight between 160 and 480, molar refractivity between 40 and 130 and total number of atoms between 20 and 70 [37]. The rules have been developed that discriminates between drug-likeness and nondrug-likeness of chemical molecules. All the compounds are found drug likeness and suitable according to MDDR like rule and rule of five respectively. The designed compounds are also qualified the CMC like rule except compound $\mathbf{4 g}$ and $\mathbf{4 l}$. The outcomes results of compounds with different rules are presented in Table 11.

Table 11: Drug likeness/Violation prediction by different rules for compounds (4a-1).

\begin{tabular}{|c|c|c|c|c|c|c|c|c|c|c|}
\hline Compd. & \multicolumn{2}{|c|}{ CMC like Rule } & \multicolumn{2}{|c|}{ Lead like Rule } & \multicolumn{2}{|c|}{ MDDR like Rule } & \multicolumn{2}{|c|}{ Rule of Five } & \multicolumn{2}{|c|}{ WDI like Rule } \\
\hline & Rule & Violation & Rule & Violation & Rule & Violation & \multicolumn{2}{|c|}{ Rule } & Violation & \multicolumn{2}{|c|}{ Rule } & Violation \\
\hline $\mathbf{4 a}$ & Qualified & 0 & Suitable & 0 & Drug like & 0 & Suitable & 0 & Out of $90 \%$ cut off & 1 \\
\hline $\mathbf{4 b}$ & Qualified & 0 & Violated & 2 & Drug like & 0 & Suitable & 0 & Out of $90 \%$ cut off & 2 \\
\hline $\mathbf{4 c}$ & Qualified & 0 & Violated & 2 & Drug like & 0 & Suitable & 0 & Out of $90 \%$ cut off & 1 \\
\hline $\mathbf{4 d}$ & Qualified & 0 & Violated & 1 & Drug like & 0 & Suitable & 0 & Out of $90 \%$ cut off & 0 \\
\hline $\mathbf{4 e}$ & Qualified & 0 & Violated & 2 & Drug like & 0 & Suitable & 0 & Out of $90 \%$ cut off & 1 \\
\hline $\mathbf{4 f}$ & Qualified & 0 & Violated & 2 & Drug like & 0 & Suitable & 0 & Out of $90 \%$ cut off & 2 \\
\hline $\mathbf{4 g}$ & Not Qualified & 1 & Violated & 2 & Drug like & 0 & Suitable & 0 & Out of $90 \%$ cut off & 2 \\
\hline $\mathbf{4 h}$ & Qualified & 0 & Violated & 1 & Drug like & 0 & Suitable & 0 & Out of $90 \%$ cut off & 1 \\
\hline $\mathbf{4 i}$ & Qualified & 0 & Violated & 2 & Drug like & 0 & Suitable & 0 & Out of $90 \%$ cut off & 1 \\
\hline $\mathbf{4 j}$ & Qualified & 0 & Violated & 1 & Drug like & 0 & Suitable & 0 & Out of $90 \%$ cut off & 1 \\
\hline $\mathbf{4 k}$ & Qualified & 0 & Violated & 2 & Drug like & 0 & Suitable & 0 & Out of $90 \%$ cut off & 1 \\
\hline $\mathbf{4 l}$ & Not Qualified & 1 & Violated & 2 & Drug like & 0 & Suitable & 0 & Out of $90 \%$ cut off & 1 \\
\hline
\end{tabular}

\subsection{Pharmacological evaluation}

All the selected compounds were submitted to National Cancer Institute (NCI), USA for evaluating their anticancer activity at the dose $1 \times 10^{-5} \mathrm{M}$ against full NCI 60 cell lines panels representing full nine human systems as leukemia, melanoma and cancers of lung, colon, brain, breast, ovary, kidney and prostate. The compounds added at a concentration $\left(1 \times 10^{-5} \mathrm{M}\right)$ and the culture incubated for $48 \mathrm{~h}$. End point determinations was made with a protein binding dye, Sulforhodamine B.
Results of each compound were reported as a mean graph of percent growth of treated cells. There after obtaining the results for one dose assay, analysis of historical Developmental Therapeutics Program (DTP) was performed and compound 4b (NCS: 759210), 4k (NCS: 761982) and 4l (NCS: 761983) satisfied pre-determined threshold inhibition criteria and obtained result are mentioned in Table 12.

Table 12: \% Growth inhibition of NCI 60 cancer cell line against the test compounds.

\begin{tabular}{|c|c|c|c|c|c|c|c|c|c|c|c|c|}
\hline \multirow[t]{2}{*}{ Panel } & Compd. $\rightarrow$ & $4 a$ & $4 b$ & $4 c$ & 4d & $4 e$ & $4 f$ & $4 g$ & $4 h$ & $4 j$ & $4 k$ & 41 \\
\hline & $\begin{array}{c}\text { Cell line } \\
\downarrow\end{array}$ & $\begin{array}{l}\text { NSC: } \\
759209\end{array}$ & $\begin{array}{l}\text { NSC: } \\
759210\end{array}$ & $\begin{array}{l}\text { NSC: } \\
759211\end{array}$ & $\begin{array}{l}\text { NSC: } \\
760445\end{array}$ & $\begin{array}{l}\text { NSC: } \\
759212\end{array}$ & $\begin{array}{l}\text { NSC: } \\
755140\end{array}$ & $\begin{array}{l}\text { NSC: } \\
755141\end{array}$ & $\begin{array}{l}\text { NSC: } \\
755142\end{array}$ & $\begin{array}{l}\text { NSC: } \\
755143\end{array}$ & $\begin{array}{l}\text { NSC: } \\
761982\end{array}$ & $\begin{array}{l}\text { NSC: } \\
761983\end{array}$ \\
\hline Leukemia & $\begin{array}{l}\text { CCRF-CEM } \\
\text { HL-60(TB) } \\
\text { K-562 } \\
\text { MOLT-4 } \\
\text { RPMI-8226 } \\
\text { SR }\end{array}$ & $\begin{array}{l}87.72 * \\
90.80 \\
82.19^{*} \\
73.39^{*} \\
76.16^{*} \\
\mathrm{Nt}\end{array}$ & $\begin{array}{l}77.62^{*} \\
84.07^{*} \\
76.34^{*} \\
55.75^{* *} \\
70.66^{*} \\
\mathrm{Nt}\end{array}$ & $\begin{array}{l}93.40 \\
78.35^{*} \\
81.71^{*} \\
78.41^{*} \\
92.97 \\
\mathrm{Nt}\end{array}$ & $\begin{array}{l}96.83 \\
99.19 \\
\mathrm{Nt} \\
102.58 \\
105.47 \\
\mathrm{Nt}\end{array}$ & $\begin{array}{l}95.27 \\
85.05^{*} \\
95.36 \\
84.81^{*} \\
100.21 \\
\mathrm{Nt}\end{array}$ & $\begin{array}{l}104.99 \\
110.13 \\
112.13 \\
96.25 \\
109.99 \\
91.40\end{array}$ & $\begin{array}{l}28.46^{* *} \\
90.58 \\
94.23 \\
75.29^{*} \\
57.38^{* *} \\
82.26^{*}\end{array}$ & $\begin{array}{l}96.41 \\
108.40 \\
104.19 \\
92.39 \\
107.56 \\
82.01 *\end{array}$ & $\begin{array}{l}101.30 \\
105.83 \\
101.20 \\
92.80 \\
117.33 \\
87.70^{*}\end{array}$ & $\begin{array}{l}100.43 \\
89.62 \\
90.58 \\
82.75 \\
95.43 \\
\mathrm{Nt}\end{array}$ & $\begin{array}{l}\mathrm{Nt} \\
80.52 * \\
79.77 * \\
8.54 * * \\
73.07 * \\
14.94 * *\end{array}$ \\
\hline $\begin{array}{l}\text { Non-Small } \\
\text { Cell Lung } \\
\text { Cancer }\end{array}$ & $\begin{array}{l}\text { A549/ATCC } \\
\text { EKVX } \\
\text { HOP-62 } \\
\text { HOP-92 } \\
\text { NCI-H226 } \\
\text { NCI-H23 } \\
\text { NCI-H322M } \\
\text { NCI-H460 } \\
\text { NCI-H522 }\end{array}$ & $\begin{array}{l}92.62 \\
64.27^{* *} \\
94.14 \\
61.48^{* *} \\
104.08 \\
86.47^{*} \\
90.00 \\
102.09 \\
79.43^{*}\end{array}$ & $\begin{array}{l}94.74 \\
63.40^{* *} \\
89.13^{*} \\
60.61^{* *} \\
106.51 \\
85.13^{*} \\
84.41^{*} \\
99.59 \\
73.95^{* *}\end{array}$ & $\begin{array}{l}102.78 \\
85.15^{*} \\
97.14 \\
70.86^{*} \\
103.80 \\
98.60 \\
87.65^{*} \\
108.45 \\
90.77\end{array}$ & $\begin{array}{l}104.19 \\
67.58^{* *} \\
106.06 \\
\mathrm{Nt} \\
89.72^{*} \\
97.36 \\
103.84 \\
104.64 \\
108.07\end{array}$ & $\begin{array}{l}115.06 \\
132.66 \\
102.07 \\
98.17 \\
109.36 \\
95.41 \\
102.21 \\
112.99 \\
98.86\end{array}$ & $\begin{array}{l}104.25 \\
106.15 \\
111.22 \\
\mathrm{Nt} \\
101.91 \\
101.45 \\
101.46 \\
115.88 \\
101.36\end{array}$ & $\begin{array}{l}98.46 \\
103.83 \\
101.38 \\
\mathrm{Nt} \\
103.65 \\
95.77 \\
110.89 \\
110.10 \\
86.25^{*}\end{array}$ & $\begin{array}{l}105.31 \\
115.97 \\
103.37 \\
\mathrm{Nt} \\
101.32 \\
101.85 \\
114.68 \\
108.30 \\
95.52\end{array}$ & $\begin{array}{l}103.34 \\
99.24 \\
102.09 \\
\mathrm{Nt} \\
115.21 \\
101.88 \\
92.89 \\
115.69 \\
98.40\end{array}$ & $\begin{array}{l}78.87 \\
45.11^{* *} \\
82.28 \\
\mathrm{Nt} \\
46.79 * * \\
68.28^{* *} \\
63.12 * * \\
90.73 \\
87.29\end{array}$ & $\begin{array}{l}63.75^{* *} \\
84.06^{*} \\
82.02^{*} \\
66.09^{* *} \\
73.72^{*} \\
74.48^{*} \\
\mathrm{Nt} \\
34.60^{* *} \\
84.17^{*}\end{array}$ \\
\hline Colon Cancer & $\begin{array}{l}\text { COLO 205 } \\
\text { HCC-2998 } \\
\text { HCT-116 } \\
\text { HCT-15 } \\
\text { HT29 } \\
\text { KM12 } \\
\text { SW-620 }\end{array}$ & $\begin{array}{l}105.45 \\
119.43 \\
82.17 * \\
107.30 \\
92.87 \\
96.72 \\
108.38\end{array}$ & $\begin{array}{l}101.62 \\
90.34 \\
73.63 * \\
97.41 \\
93.43 \\
99.77 \\
103.19\end{array}$ & $\begin{array}{l}102.35 \\
137.83 \\
97.63 \\
99.24 \\
99.89 \\
104.25 \\
110.30\end{array}$ & $\begin{array}{l}\mathrm{Nt} \\
113.38 \\
102.52 \\
104.54 \\
106.40 \\
106.41 \\
102.17\end{array}$ & $\begin{array}{l}102.60 \\
102.03 \\
98.03 \\
100.31 \\
109.17 \\
105.69 \\
107.04\end{array}$ & $\begin{array}{l}113.69 \\
107.23 \\
121.84 \\
99.55 \\
\mathrm{Nt} \\
117.54 \\
109.14\end{array}$ & $\begin{array}{l}112.51 \\
104.56 \\
107.74 \\
106.85 \\
\mathrm{Nt} \\
104.42 \\
103.13\end{array}$ & $\begin{array}{l}124.25 \\
111.83 \\
110.74 \\
101.90 \\
\mathrm{Nt} \\
113.71 \\
113.16\end{array}$ & $\begin{array}{l}119.33 \\
107.62 \\
105.50 \\
100.24 \\
\mathrm{Nt} \\
111.44 \\
108.71\end{array}$ & $\begin{array}{l}132.71 \\
127.87 \\
93.75 \\
34.71 * * \\
91.57 \\
95.02 * * \\
71.07\end{array}$ & $\begin{array}{l}103.12 \\
79.80^{*} \\
63.80^{* *} \\
89.25^{*} \\
89.51^{*} \\
84.39^{*} \\
83.48^{*}\end{array}$ \\
\hline CNS Cancer & $\begin{array}{l}\text { SF-268 } \\
\text { SF-295 } \\
\text { SF-539 } \\
\text { SNB-19 } \\
\text { SNB-75 } \\
\text { U251 }\end{array}$ & $\begin{array}{l}107.94 \\
100.52 \\
98.84 \\
101.14 \\
92.63 \\
90.92\end{array}$ & $\begin{array}{l}93.57 \\
95.65 \\
97.47 \\
92.67 \\
86.92 * \\
94.87\end{array}$ & $\begin{array}{l}100.85 \\
105.07 \\
116.23 \\
100.83 \\
84.59 * \\
105.84\end{array}$ & $\begin{array}{l}102.29 \\
102.41 \\
99.26 \\
102.15 \\
71.91^{*} \\
96.08\end{array}$ & $\begin{array}{l}109.10 \\
115.69 \\
102.22 \\
101.33 \\
101.49 \\
106.75\end{array}$ & $\begin{array}{l}117.80 \\
109.89 \\
98.83 \\
100.20 \\
89.77 * \\
102.56\end{array}$ & $\begin{array}{l}104.36 \\
125.48 \\
92.56 \\
92.95 \\
96.08 \\
99.39\end{array}$ & $\begin{array}{l}105.04 \\
102.92 \\
103.33 \\
110.96 \\
99.11 \\
103.41\end{array}$ & $\begin{array}{l}111.14 \\
111.23 \\
100.30 \\
108.21 \\
97.88 \\
109.90\end{array}$ & $\begin{array}{l}63.37 * * \\
78.53 \\
115.22 \\
66.82 \\
132.99 \\
83.35\end{array}$ & $\begin{array}{l}95.85 \\
79.75^{*} \\
86.76^{*} \\
90.97 \\
67.51^{* *} \\
85.55^{*}\end{array}$ \\
\hline
\end{tabular}




\begin{tabular}{|c|c|c|c|c|c|c|c|c|c|c|c|c|}
\hline Melanoma & $\begin{array}{l}\text { LOX IMVI } \\
\text { MALME-3M } \\
\text { M14 } \\
\text { MDA-MB-435 } \\
\text { SK-MEL-2 } \\
\text { SK-MEL-28 } \\
\text { SK-MEL-5 } \\
\text { UACC-257 } \\
\text { UACC-62 }\end{array}$ & $\begin{array}{l}92.90 \\
99.90 \\
102.96 \\
101.82 \\
96.82 \\
108.42 \\
100.49 \\
101.31 \\
76.58^{*}\end{array}$ & $\begin{array}{l}80.32 * \\
94.26 \\
101.96 \\
94.32 \\
88.65 \\
112.03 \\
99.41 \\
103.00 \\
69.38 * \\
\end{array}$ & $\begin{array}{l}95.97 \\
98.34 \\
98.03 \\
105.00 \\
104.28 \\
110.50 \\
105.38 \\
111.69 \\
89.91 * \\
\end{array}$ & $\begin{array}{l}94.67 \\
112.33 \\
106.02 \\
102.36 \\
103.41 \\
106.45 \\
96.70 \\
104.28 \\
97.24 \\
\end{array}$ & $\begin{array}{l}101.05 \\
94.16 \\
98.95 \\
108.82 \\
108.88 \\
107.74 \\
107.42 \\
111.48 \\
100.64\end{array}$ & $\begin{array}{l}88.61 * \\
93.56 \\
129.33 \\
111.61 \\
\mathrm{Nt} \\
112.86 \\
104.97 \\
109.21 \\
105.18 \\
\end{array}$ & $\begin{array}{l}67.52 * * \\
96.33 \\
100.58 \\
76.71 * \\
\mathrm{Nt} \\
105.02 \\
99.75 \\
113.54 \\
78.94 * \\
\end{array}$ & $\begin{array}{l}94.44 \\
92.00 \\
111.16 \\
102.72 \\
\mathrm{Nt} \\
105.40 \\
105.41 \\
111.94 \\
97.94 \\
\end{array}$ & $\begin{array}{l}90.45 \\
77.82 * \\
104.85 \\
111.29 \\
\quad \mathrm{Nt} \\
116.12 \\
104.12 \\
113.59 \\
91.04 \\
\end{array}$ & $\begin{array}{l}64.41 * * \\
52.15^{* *} \\
116.65 \\
145.79 \\
74.78 \\
59.14 \\
86.78 \\
18.60^{* *} \\
58.95^{* *}\end{array}$ & $\begin{array}{l}53.93 * * \\
90.91 \\
89.84 * \\
91.89 \\
100.33 \\
104.24 \\
68.87 * \\
101.20 \\
66.22 * *\end{array}$ \\
\hline $\begin{array}{l}\text { Ovarian } \\
\text { Cancer }\end{array}$ & $\begin{array}{l}\text { IGROV1 } \\
\text { OVCAR-3 } \\
\text { OVCAR-4 } \\
\text { OVCAR-5 } \\
\text { OVCAR-8 } \\
\text { NCI/ADR-RES } \\
\text { SK-OV-3 }\end{array}$ & $\begin{array}{l}82.61 * \\
106.45 \\
94.07 \\
103.08 \\
93.64 \\
92.55 \\
100.21 \\
\end{array}$ & $\begin{array}{l}74.58^{*} \\
95.04 \\
82.93^{*} \\
107.85 \\
88.35^{*} \\
91.44 \\
96.16 \\
\end{array}$ & $\begin{array}{l}90.46 \\
117.74 \\
104.50 \\
111.87 \\
101.42 \\
110.54 \\
97.57\end{array}$ & $\begin{array}{l}91.31 \\
102.05 \\
104.98 \\
110.86 \\
101.60 \\
105.98 \\
102.98\end{array}$ & $\begin{array}{l}99.70 \\
117.54 \\
104.43 \\
105.71 \\
108.05 \\
103.53 \\
104.86\end{array}$ & $\begin{array}{l}104.33 \\
124.13 \\
104.79 \\
92.23 \\
110.39 \\
98.58 \\
113.63\end{array}$ & $\begin{array}{l}123.92 \\
121.00 \\
100.38 \\
88.73^{*} \\
105.39 \\
97.04 \\
114.42 \\
\end{array}$ & $\begin{array}{l}117.72 \\
122.41 \\
110.84 \\
106.54 \\
106.87 \\
105.29 \\
116.12\end{array}$ & $\begin{array}{l}105.89 \\
117.84 \\
106.52 \\
102.86 \\
104.73 \\
99.80 \\
107.81\end{array}$ & $\begin{array}{l}52.18^{* *} \\
114.80 \\
86.20 \\
\mathrm{Nt} \\
78.97 \\
9.30^{* *} \\
107.95\end{array}$ & $\begin{array}{l}65.32 * * \\
101.59 \\
\mathrm{Nt} \\
97.97 \\
88.49 \\
86.11 \\
98.72\end{array}$ \\
\hline $\begin{array}{l}\text { Renal } \\
\text { Cancer }\end{array}$ & $\begin{array}{l}786-0 \\
\text { A498 } \\
\text { ACHN } \\
\text { CAKI-1 } \\
\text { RXF 393 } \\
\text { SN12C } \\
\text { TK-10 } \\
\text { UO-31 } \\
\end{array}$ & $\begin{array}{l}101.54 \\
103.39 \\
103.03 \\
81.27 * \\
117.10 \\
85.50 * \\
112.01 \\
72.01 * *\end{array}$ & $\begin{array}{l}100.14 \\
80.48^{*} \\
108.21 \\
84.08^{*} \\
113.43 \\
86.86^{*} \\
117.03 \\
61.32^{* *}\end{array}$ & $\begin{array}{l}105.07 \\
105.86 \\
89.77^{*} \\
92.93 \\
125.13 \\
87.25^{*} \\
121.38 \\
68.81^{*}\end{array}$ & $\begin{array}{l}108.02 \\
105.83 \\
101.30 \\
90.21 \\
111.73 \\
96.08 \\
148.14 \\
83.60^{*}\end{array}$ & $\begin{array}{l}103.46 \\
101.91 \\
100.41 \\
106.78 \\
128.11 \\
100.05 \\
114.19 \\
83.36^{*}\end{array}$ & $\begin{array}{l}101.93 \\
99.94 \\
105.35 \\
97.21 \\
115.45 \\
94.39 \\
109.10 \\
84.84 * \\
\end{array}$ & $\begin{array}{l}81.10^{*} \\
93.24 \\
100.72 \\
97.44 \\
99.90 \\
86.84^{*} \\
100.05 \\
86.26^{*} \\
\end{array}$ & $\begin{array}{l}103.76 \\
96.03 \\
112.71 \\
101.66 \\
121.29 \\
92.60 \\
107.92 \\
92.36 \\
\end{array}$ & $\begin{array}{l}105.16 \\
100.86 \\
106.71 \\
109.82 \\
123.77 \\
98.54 \\
109.98 \\
91.95 \\
\end{array}$ & $\begin{array}{c}71.26 \\
88.46 \\
38.88^{* *} \\
36.53^{* *} \\
107.83 \\
69.75^{* *} \\
35.89^{* *} \\
55.85^{* *} \\
\end{array}$ & $\begin{array}{l}97.21 \\
61.74 * * \\
87.01 * \\
82.78 * \\
98.48 \\
87.14 * \\
100.43 \\
44.17 * * \\
\end{array}$ \\
\hline $\begin{array}{l}\text { Prostate } \\
\text { Cancer }\end{array}$ & $\begin{array}{l}\text { PC-3 } \\
\text { DU-145 }\end{array}$ & $\begin{array}{l}79.94 * \\
110.17\end{array}$ & $\begin{array}{l}76.32 * \\
103.88 \\
\end{array}$ & $\begin{array}{l}78.91 * \\
112.54\end{array}$ & $\begin{array}{l}96.38 \\
\mathrm{Nt}\end{array}$ & $\begin{array}{l}92.11 \\
113.19 \\
\end{array}$ & $\begin{array}{l}\mathrm{Nt} \\
116.71 \\
\end{array}$ & $\begin{array}{l}\mathrm{Nt} \\
110.59 \\
\end{array}$ & $\begin{array}{l}\mathrm{Nt} \\
108.53 \\
\end{array}$ & $\begin{array}{l}\mathrm{Nt} \\
108.35\end{array}$ & $\begin{array}{l}80.70 \\
135.32 \\
\end{array}$ & $\begin{array}{l}77.04 * \\
98.38 \\
\end{array}$ \\
\hline $\begin{array}{l}\text { Breast } \\
\text { Cancer }\end{array}$ & $\begin{array}{l}\text { MCF7 } \\
\text { MDA-MB- } \\
\text { 231/ATCC } \\
\text { HS 578T } \\
\text { BT-549 } \\
\text { T-47D } \\
\text { MDA-MB-468 }\end{array}$ & $\begin{array}{l}84.29 * \\
76.05^{*} \\
\\
95.94 \\
87.10^{*} \\
79.11 * \\
104.54 \\
\end{array}$ & $\begin{array}{l}76.01 * \\
75.32 * \\
\\
92.26 \\
84.83^{*} \\
74.37^{*} \\
89.05^{*}\end{array}$ & $\begin{array}{l}84.59^{*} \\
80.26^{*} \\
\\
104.42 \\
103.89 \\
83.93 * \\
118.77 \\
\end{array}$ & $\begin{array}{l}82.77 * \\
90.35 \\
\\
\mathrm{Nt} \\
91.65 \\
88.76 \\
95.51\end{array}$ & $\begin{array}{l}92.76 \\
91.94 \\
\\
109.68 \\
97.76 \\
101.25 \\
120.64\end{array}$ & \begin{tabular}{l|l|}
94.33 \\
111.18 \\
\\
109.36 \\
116.61 \\
108.68 \\
112.87 \\
\end{tabular} & $\begin{array}{l}64.24^{* *} \\
93.85 \\
\\
100.25 \\
101.02 \\
81.56^{*} \\
106.20 \\
\end{array}$ & $\begin{array}{l}91.03 \\
98.71 \\
\\
126.78 \\
111.51 \\
108.06 \\
103.62\end{array}$ & $\begin{array}{l}105.96 \\
89.67 * \\
\\
111.82 \\
113.54 \\
102.27 \\
108.19 \\
\end{array}$ & $\begin{array}{l}90.56 \\
73.95 \\
\\
89.85 \\
81.14 \\
65.66^{* *} \\
101.54 \\
\end{array}$ & $\begin{array}{l}35.66 * * \\
87.25 * \\
\\
81.30 * \\
74.46 * \\
71.58 * \\
79.03 * \\
\end{array}$ \\
\hline \multicolumn{2}{|c|}{ Mean } & 93.83 & 88.91 & 99.18 & 100.53 & 103.89 & 106.04 & 96.09 & 105.73 & 104.78 & 20.03 & 78.75 \\
\hline \multicolumn{2}{|c|}{ Delta } & 32.35 & 33.16 & 30.37 & 32.95 & 20.53 & 21.20 & 67.63 & 23.72 & 26.96 & 22.01 & 70.21 \\
\hline \multicolumn{2}{|c|}{ Range } & 57.95 & 61.28 & 69.02 & 80.56 & 49.30 & 44.49 & 97.02 & 44.77 & 45.95 & 51.21 & 95.70 \\
\hline
\end{tabular}

*good anticancer activity, ** significant active for particular cell line.

\section{CONCLUSION}

The series of 1-(1H-benzo[ $[$ ] imidazol-2-yl)-3-(5-(methyl substituted)-1,3,4oxadiazol-2-yl) propan-1-one (4a-1) with the secondary amine was synthesized under microwave irradiation in good yields. FT-IR, ${ }^{1} \mathrm{H}-\mathrm{NMR},{ }^{13} \mathrm{C}-\mathrm{NMR}$, Mass Spectroscopy and Elemental analysis were used for the spectral characterization of synthetic compounds. The compounds were evaluated for their anticancer activity against full NCI 60 cell lines panel. The screening data obtained from $\mathrm{NCI}$ indicated that the compounds $\mathbf{4 b}, \mathbf{4 k}$ and $\mathbf{4 l}$ exhibited good percentage growth inhibition against human cancer cell lines. Oral bioavailability, molecular descriptors, drug likeness properties and bioactivity was estimated as per the Lipinski rules of five and results outcomes of compounds showed good oral bioavailability. Drug relevant properties was predicted by OSIRIS property explorer and results indicates that compounds get good drug score but compound $\mathbf{4 a}, \mathbf{4} \mathbf{c}$ and $\mathbf{4 k}$ get excellent drug likeness and compounds $4 \mathbf{c}, 4 \mathbf{k}$ and $4 \mathbf{i}$ get excellent drug score.

The acute toxicity of compounds was evaluated by T.E.S.T program (US-EPA) in form of LD50 and mutagenicity. The synthetic compounds were found to have a high degree of lipophilicity, which designates a good lipid solubility that helps the drug to interact with membranes. The compounds are found substrate for CYP 450 3A4 except compound $\mathbf{4 l}$ and non substrate for CYP 450 2D6 except compound $\mathbf{4 a}$ and $\mathbf{4} \mathbf{c}$ but specially compounds $\mathbf{4 h}-\mathbf{4 j}$ are found inhibitor of CYP 450 2C19, CYP 450 3A4 and CYP 450 2C9. Compound $\mathbf{4 c}$ was exhibited higher drug score, bioactivity score and revealed good drug relevant properties, ADME and no toxicity profile in compared to other compounds. The most active compound of the series was found $\mathbf{4 c}$ and $\mathbf{4 k}$ therefore further studies on this compound continue in our research laboratory to acquire more information about SAR and QSAR.

\section{Conflict of Interest}

The authors declare no conflict of interest, financial or otherwise.

\section{ACKNOWLEDGMENTS}

Authors are grateful to Project Manager, Developmental Therapeutics Program (DTP), National Cancer Institute (NCI), Chemotherapeutic Agents Repository, Fisher Bio Services, Rockville, MD, USA for anticancer screening of compounds and authors are also thank full to Prince Sattam Bin Abdulaziz University for providing the resources to conduct the research.

\section{REFERENCES}

1. Satoh M, Cherian MG (1994) Modulation of resistance to anticancer drugs by inhibition of metallothionein synthesis. Imura Cancer Res 54: 5255.

2. Zhang K, Mack T, Kim PW (1998) Inhibitors of Multidrug Resistance to Antitumor Agents. Int J Oncol 12: 971.

3. Verweij J, Dejonge MJA (2000) Achievements and future of chemotherapy. Eur J Cancer 36: 1479.

4. Volm M (1998) Multidrug resistance and its reversal. Anticancer Res 18: 2905.

5. Vita VT, Hellman S, Rosenberg SA (1992) Cancer principle practical oncology. 4th ed. Philadelphia, J.B. Lippincott, Co., USA.

6. Hartwell LH, Kashan MB (1994) Cell cycle control and cancer. Science 266: 1821.

7. Li Y, Tan C, Gao C, Zhang C, Luan X, Chen X, Liu H, Chen Y, Jiang Y (2011) Discovery of benzimidazole derivatives as novel multi-target EGFR, VEGFR-2 and PDGFR kinase inhibitors. Bioorg Med Chem 19: 4529.

8. Demirayak S, Kayagil I, Yurttas L (2011) Microwave supported synthesis of some novel 1,3-diarylpyrazino[1,2-a]benzimidazole derivatives and investigation of their anticancer activities. Eur J Med Chem 46: 411.

9. Husain A, Rashid M, Siddiqui AA, Mishra R (2013) Benzimidazole clubbed with triazolo-thiadiazoles and triazolo-thiadiazines: New anticancer agents. Eur J Med Chem 62: 785-798.

10. Husain A, Rashid M, Mishra R, Praveen S, Shin DS, Kumar D (2012) Benzimidazole bearing oxadiazole and triazolo-thiadiazoles nucleus: Design and synthesis as anticancer agents. Bioorg Med Chem Lett 22: 5438-5444. 
11. Sondhi SM, Rani R, Singh J, Roy P, Agrawal SK, Saxena AK (2010) Solvent free synthesis, anti-inflammatory and anticancer activity evaluation of tricyclic and tetracyclic benzimidazole derivatives. Bioorg Med Chem Lett 20: 2306.

12. Penning TD, Zhu GD, Gandhi VB, Gong J, Liu X, Shi Y, Klinghoffer V, Johnson EF, Donawho CK, Frost DJ, DiazBouska VB, Osterling DJ, Olson AM, Marsh KC, Luo Y, Giranda VL (2009) Discovery of the Poly(ADPribose) polymerase (PARP) inhibitor 2-[(R)-2-methylpyrrolidin-2-yl]-1Hbenzimidazole-4-carboxamide (ABT-888) for the treatment of cancer. J Med Chem 52: 514

13. Rashid M, Husain A, Mishra R (2012) Synthesis of benzimidazoles bearing oxadiazole nucleus as anticancer agents. Eur J Med Chem 54: 855-866.

14. Rashid M, Husain A, Mishra R, Karim S, Khan S, Ahmad M, Al-wabel N, Husain A, Ahmad A, Khan SA (2019) Design and synthesis of benzimidazoles containing substituted oxadiazole, thiadiazole and triazolothiadiazines as a source of new anticancer agents. Arab J Chem 12(8) 3202-3224

15. Rashid M, Husain A, Shaharyar M, Sarafroz M (2014) Anticancer Activity of New Compounds Using Benzimidazole as a Scaffold. Anti-Cancer Agents in Med Chem 14: 1003-1018.

16. Husain A, Varshney MM, Rashid M, Mishra R, Akhter A (2011) Benzimidazole: A Valuable Insight in to the Recent Advances and Biological Activities. J Pharm Res 4: 413-419.

17. Lio SC, Johnson J, Chatterjee A, Ludwig JW, Millis D, Banie H, Sircar JC Sinha A, Richards ML (2008) Disruption of golgi processing by 2-phenyl benzimidazole analogs blocks cell proliferation and slows tumor growth. Can Chemo Pharmaco 61: 1045

18. Goker H, Kus C, Boykin DW, Yldz S, Altanlar N (2002) Synthesis of some new 2-substituted-phenyl-1H-benzimidazole-5-carbonitriles and their potent activity against Candida species. Bioorg Med Chem 10: 2589.

19. Kerimov I, Kilcigil GA, Eke BC, Altanlar N (2007) Synthesis, antifungal and antioxidant screening of some novel benzimidazole derivatives. J Enzyme Inhib Med 17: 696.

20. Sharma D, Narasimhan B, Kumar P, Judge V, Narang R, Clereq ED, Balzarini J (2009) Synthesis, antimicrobial and antiviral activity of substituted benzimidazoles. J Enzyme Inhib Med 24: 1161.

21. Kumar BVS, Vaidya SD, Kumar RV, Bhirud SB, Mane RB (2006) Biological activity evaluation of novel $\mathrm{n}$-heterocyclic carbene precursors. Eur J Med Chem 41: 599

22. Francisca P, Helgi JC, Jaime PV, Juan CP, Sergio RM, Guadalupe PH, Nayeli LB, Alicia HC, Rafael C, Francisco HL (2009) Synthesis and in-vitro cysticidal activity of new benzimidazole derivatives. Eur J Med Chem 44: 1794.

23. Padmavathi V, Reddy SG, Padmaja A, Kondaiah P, Shazia A (2009) Synthesis, antimicrobial and cytotoxic activities of 1,3,4-oxadiazoles, 1,3,4thiadiazoles and 1,2,4-triazoles. Eur J Med Chem 44: 2106.

24. El-Sayed NS, El-Bendary ER, El-Ashry SM, El-Kerdawy MM (2011) Synthesis and antitumor activity of new sulfonamide derivatives of thiadiazolo[3,2-a]pyrimidines. Eur J Med Chem 46: 3714

25. Formagio ASN, Tonin LTD, Foglio MA, Madjarof C, Carvalho JE, Costa WFD, Cardoso FP, Sarragiotto MH (2008) Synthesis of pyrrolo [2, 1-c] [1, 4] benzodiazepines. Bioorg Med Chem 16: 9660.

26. Matysiak J (2007) Evaluation of electronic, lipophilic and membrane affinity effects on antiproliferative activity of 5-substituted-2-(2,4-dihydroxyphenyl)1,3,4-thiadiazoles against various human cancer cells. Eur J Med Chem 42: 940.

27. Lissitchkov T, Arnaudov G, Peytchev D, Merkle KJ (2006) Phase-I/II study to evaluate dose limiting toxicity, maximum tolerated dose, and tolerability of bendamustine $\mathrm{HCl}$ in pre-treated patients with $\mathrm{B}$-chronic lymphocytic leukaemia (Binet stages B and C) requiring therapy. Canc Res Clin Onco 132: 99.

28. Knauf WU, Lissichkov T, Aldaoud A (2009) Phase III randomized study of bendamustine compared with chlorambucil in previously untreated patients with chronic lymphocytic leukemia. J Clin Onco 27: 4378.

29. Dalip K, Gautam P, Angela K, Chavers Kuei HC, Kavita S (2011) Synthesis of novel 1,2,4-oxadiazoles and analogues as potential anticancer agents. Eur J Med Chem 46: 3085-3098.

30. Maria CT, Asra M, Xiaohu O, David S, James F, Stan M, Margarita C, Bridget F, Jacqueline D. Eugene LP, Chekler PK, James RT (2010) Antitumor Activity of IMC-038525, a Novel Oral Tubulin Polymerization Inhibitor. Im Clon Systems. Inc. New York, USA, 3: 318.

31. Tolner B, Hartley JA, Hochhauser D (2001) Transcriptional regulation of topoisomerase II alpha at confluence and pharmacological modulation of expression by bis-benzimidazole drugs. Mol Pharmacol 59: 699 .

32. Duanmu C, Shahrik LK, Holly HH, Hamel E (1989) Tubulin-dependent hydrolysis of guanosine triphosphate as a screening test to identify new antitubulin compounds with potential as antimitotic agents: application to carbamates of aromatic amines. Cancer Res 49: 1344-1348.
33. Lipinski CA, Lombardo F, Dominy BW, Feeney PJ (1997) Experimental and computational approaches to estimate solubility and permeability in drug discovery and development settings. Adv Drug Deliver Rev 23: 4-25.

34. Ertl P, Rohde B, Selzer P (2000) Fast calculation of molecular polar surface area as a sum of fragment-based contributions and its application to the prediction of drug transport properties. J Med Chem 43: 3714-3717.

35. Molinspiration software (www.molinspiration.com/cgi-bin/properties)

36. Teague SJ, Davis AM, Leeson PD, Oprea T, Angew (1999) The Design of Lead like Combinatorial Libraries. Chem Int Ed 38: 3743.

37. Ghose AK, Viswanadhan VN, Wendoloski JJ (1999) A knowledge-based approach in designing combinatorial or medicinal chemistry libraries for drug discovery; A qualitative and quantitative characterization of known drug databases. J Comb Chem 1: 55.

38. Oprea TI (2000) Property distribution of drug-related chemical databases. J Comp Aided Mol Des 14: 251.

39. Kulkarni A, Han Y, Hopfinger AJJ (2002) Predicting Caco-2 cell permeation coefficients of organic molecules using membrane-interaction QSAR analysis, Chem. Inf Comput Sci 42: 331 .

40. Veber DF, Johnson SR, Cheng HY, Smith BR, Ward KW, Kopple KD (2002) Molecular properties that influence the oral bioavailability of drug candidates. J Med Chem 45: 2615-2623.

41. Bhutani R, Pathak DP, Kapoor G, Husain A, Iqbal MA (2019) Novel hybrids of benzothiazole-1,3,4-oxadiazole-4-thiazolidinone: Synthesis, in-silico ADME study, molecular docking and in-vivo anti-diabetic assessment. Bioorg Chem 83: 6-19.

42. Faizia M, Jahania R, Ebadib SA, Tabatabaic SA, Rezaeec E, Lotfalieic M, Aminid M, Almasirad A (2017) Novel 4-thiazolidinone derivatives as agonists of benzodiazepine receptors: design, synthesis and pharmacological evaluation. EXCLI J 16: 52-62.

43. Kapoor G, Pathak DP, Bhutani R, Husain A, Jain S, Iqbal MA (2019) Synthesis, ADME, docking studies and in-vivo anti-hyperglycaemic potential estimation of novel Schiff base derivatives from octadec-9-enoic acid. Bioorg Chem 84: 478-492.

44. Husain A, Ahmad A, Khan SA, Asif M, Bhutani R, Al-Abbasi FA (2016) Synthesis, molecular properties, toxicity and biological evaluation of some new substituted imidazolidine derivatives in search of potent antiinflammatory agents. Saudi Pharm J 24: 104-114.

45. Asif M, Acharya M, Lakshmayya Singh A (2015) In-silico physicochemical bioactivities and toxicities prediction of 3-chloro-6-arylpyridazines and 6aryl- 4,5-dihydropyridazine-3(2H)-thiones having antitubercular activity. RGUHS J Pharm Sci 5: 81-87.

46. Namachivayam B, Raj JS, Kandakatla N (2014) 2D, 3D-QSAR, docking and optimization of 5-substituted-1H-Indazole as inhibitors of GSK3 $\beta$. Int J Pharm Pharm Sci 6: 1-8.

47. Hassan M, Ashraf Z, Abbas Q, Raza H (2018) Exploration of novel human tyrosinase inhibitors by molecular modeling, docking and simulation studies. Interdisciplinary Sci 3: 234.

48. Bhat AR, (2018) Petra, Osiris and Molinspiration: A computational bioinformatic platform for experimental in-vitro antibacterial activity of annulated uracil derivatives. Iranian Chemical Commun 7: 234.

49. Jamuna S, Rathinavel AK, Sadullah S, Sadullah M, Devaraj S (2018) In-silico approach to study the metabolism and biological activities of oligomeric proanthocyanidin complexes. Indian J Pharmacol 50: 242-250.

50. Joshi A, Kumar R, Sharma A (2018) Molecular docking studies, bioactivity score prediction, drug likeness analysis of gsk- $3 \beta$ inhibitors: a target protein involved in alzheimer's disease. Biosci Biotech Res Asia 15:562-573.

51. Kumar SA, lakshmi NR, Priya SB, latha, BH, Megalai PM (2018) In-silico design, docking, and synthesis of 3-hydroxy-3-methylglutaryl-coenzyme A reductase inhibitors.Drug Inven. Today 10 (12): 2568-2581.

52. Molecular property explorer, OSIRIS properties [internet], 2014 [cited 2014 Nov 26], Available from http://www.organic-chemistry.org/prog/peo/drugscore.

53. Thomas S (2001) Actelion's property explorer, Actelion's Pharmaceuticals Ltd., Gewer bestrasse, 16: 4123 Allschwil, Switzerland.

54. Balakrishnan N, Raj JS, Kandakatla N (2015) In-silico studies on new indazole derivatives as Gsk-3 $\beta$ inhibitors. Int J Pharm Pharm Sci 7: 295-299.

55. Rashid M (2020) Design, synthesis and ADMET prediction of bisbenzimidazole as anticanceragent. Bioorg. Chem. 96: 103576.

56. Jagadish PC, Soni N, Verma A (2013) Design, synthesis and in-vitro antioxidant activity of 1,3,5-trisubstituted-2-pyrazolines derivatives. J Chem 4: $1-7$.

57. Proudfoot JR (2002) Drugs, leads and drug-likeness: an analysis of some recently launched drugs. Bioorg Med Chem Lett 12(12): 1647-50.

58. Parua S, Sikari R, Singha S, Chakraborty G, Mondal R, Paul ND (2018) Accessing Polysubstituted Quinazolines via Nickel Catalyzed Acceptorless Dehydrogenative Coupling. J Org Chem 83: 11154-11166. 
59. Caterina MC, Perillo IA, Boiani L, Pezaroglo H, Cerecetto H, Gonzalez M, Salerno A (2008) Imidazolidines as new anti-trypanosoma cruzi agents: biological evaluation and structureactivity relationships. Bioorg Med Chem 16: 2226-2234.

60. He T, Shi R, Gong Y, Jiang G, Liu M, Qian S, Wang Z (2016) Base-Promoted Cascade Approach for the preparation of reduced knoevenagel adducts using hantzsch esters as reducing agent in water. synlett 27: 1864-1869.

61. Lee SK, Chang GS, Lee IH, Chung JE, Sung KY, No KT (2004) The PreADME: Pc-Based program for batch prediction of ADME properties. Euro QSAR 9.5-10, Istanbul, Turkey.

62. Albert LP (2001) Screening for human ADME/Tox drug properties in drug discovery. DDT 6: 357-366.

63. Lee SK, Lee IH, Kim HJ, Chang GS, Chung JE, No KT (2003) The PreADME Approach: web-based program for rapid prediction of physicochemical, drug absorption and drug-like properties. Euro QSAR 2002 designing drugs and crop protectants: processes, problems and solutions, black well publishing, Massachusetts, USA, 418-420.

64. Ames test (2012, May 12). In Wikipedia, the Free Encyclopedia. Retrieved 14:44, May 21, 2012, fromhttp://en.wikipedia.org/w/index.php?title=Ames test\&oldid=492194122.

65. Zhang L, Brett CM, Giacomini KM (1998) Hayes' principles and methods of toxicology. Ann Rev Pharma Tox 38: 431.

66. Pradhan S, Mondal S, Sinha C (2016) In search of Tuberculosis drug design: An in-silico approach to azoimidazolyl derivatives as antagonist for Cytochrome P450. J Indian Chem Soc 93: 1-18.

67. Md. Sarfaraj H, Faizul A, Hanan AE, Ismail A, Jamal AM, Jamal MD, Hend I, Mohammed A, Muhammad A, Anzarul H (2020) Anti-inflammatory, analgesic and molecular docking studies of Lanostanoic acid 3-O-a-Dglycopyranoside isolated from Helichrysum stoechas. Arab J Chem 13: 9196-9206.
68. Martin, T., (2016) Toxicity estimation software tool (TEST), US Environmental Protection Agency, Washington DC.

69. Sripriya N, Ranjith KM, Ashwin KN, Bhuvaneswari S, Udaya PNK (2019) In silico evaluation of multispecies toxicity of natural compounds. Drug Chem Toxicol. 21: 1-7.

70. Monks A, Scudiero D, Skehan P, Shoemaker R, Paull K, Vistica D, Hose C, Langley J, Cronise P (1991) Molecular targets in the National Cancer Institute drug screen. J Natl Cancer Inst 83: 757-766.

71. Skehan P, Storeng R, Scudiero D, Monks A, Mcmahon J, Vistica D, Warren JR, Bokesch H, Kenney S, Boyd MR (1990) Anticancer Activity of Novel Daphnane Diterpenoids from Daphne genkwa through Cell-Cycle Arrest and Suppression of Akt/STAT/Src Signalings in Human Lung Cancer Cells. J Natl Cancer Inst 82: 1107-1112

72. Grever MR, Schepartz SA, Chabner BA (1992) The National Cancer Institute: cancer drug discovery and development program. Semin Oncol 19: 622-638.

73. [Boyd MR, Paull KD (1995) The NCI in-vitro anticancer drug discovery screen. Drug Dev Res 34: 91-109.

74. Lin JH, Yamazaki M (2003) Role of P-glycoprotein in pharmacokinetics; clinical implications. Clinic Pharmaco 42: 59.

75. Ames BN, Gurney EG, Miller JA, Bartsch, H (1972) Carcinogens as frame shift mutagens: metabolites and derivatives of 2-acetylaminofluorene and other aromatic amine carcinogens. Proc Nat Acad Sci 69: 3128-3132.

76. Ames BN, Gurney, EG, Miller JA, Bartsch, H (1973) Carcinogens as frame shift mutagens: metabolites and derivatives of 2-acetylaminofluorene and other aromatic amine carcinogens. PNAS 69: 3128-213.

77. Peter EJ (1999) Maurice Dickins, Advances in in-vitro drug metabolism screening. PSTT 2: 13-19. 